\title{
Capturing the multivariate extremal index: bounds and interconnections
}

\author{
Andree Ehlert • Martin Schlather
}

Received: 7 February 2007 / Revised: 7 February 2008 / Accepted: 3 April 2008 / Published online: 14 May 2008

(C) The Author(s) 2008

\begin{abstract}
The multivariate extremal index function is a direction specific extension of the well-known univariate extremal index. Since statistical inference on this function is difficult it is desirable to have a broad characterization of its attributes. We extend the set of common properties of the multivariate extremal index function and derive sharp bounds for the entire function given only marginal dependence. Our results correspond to certain restrictions on the two dependence functions defining the multivariate extremal index, which are opposed to Smith and Weissman's (1996) conjecture on arbitrary dependence functions. We show further how another popular dependence measure, the extremal coefficient, is closely related to the multivariate extremal index. Thus, given the value of the former it turns out that the above bounds may be improved substantially. Conversely, we specify improved bounds for the extremal coefficient itself that capitalize on marginal dependence, thereby approximating two views of dependence that have frequently been treated separately. Our results are completed with example processes.
\end{abstract}

Keywords Multivariate extremal index function - Dependence function • Adjusted extremal coefficient • Max-stable process • Upper bound • Lower bound

AMS 2000 Subject Classifications Primary 60G70 • Secondary 60G10 • $62 \mathrm{H} 05 \cdot 62 \mathrm{H} 20$

\footnotetext{
A. Ehlert · M. Schlather $(\varangle)$

Institut für Mathematische Stochastik, Universität Göttingen,

Maschmühlenweg 8-10, 37073 Göttingen, Germany

e-mail: schlather@math.uni-goettingen.de
}

A. Ehlert
e-mail: ehlert@math.uni-goettingen.de 


\section{Introduction}

A core question in extreme value theory concentrates on suitable conditions such that the well-known results for the limiting distributions of maxima of i.i.d. random sequences can be applied to stationary random sequences. In addition to the discussion of various mixing conditions a single number known as the extremal index has become the key parameter to capture the effect of temporal dependence on the limiting distribution of maxima. An intuitive interpretation of the extremal index emphasizing its relevancy to practice is based on its reciprocal value which corresponds to the mean cluster size of extremes of the sequence (Leadbetter 1983). We are concerned with a multivariate generalization of this concept.

Then, the corresponding interpretation for the multivariate extremal index is the reciprocal average cluster size of a univariate sequence that, for each point in time, is given as the maximum of the weighted marginal sequences (Smith and Weissman 1996). That is, the multivariate extremal index is a function of weights comprising each of the respective univariate extremal indices as a special case. However, the average cluster size for arbitrary weights can, in general, not be determined by knowledge of the univariate extremal indices alone. Given the latter, the behavior of valid multivariate extremal index functions is therefore a matter of interest.

To be specific, we consider $D$-variate, stationary max-stable processes $\boldsymbol{Y}=$ $\left\{\boldsymbol{Y}_{i}=\left(Y_{i, 1}, \ldots, Y_{i, D}\right), i \in \mathbb{Z}\right\}$ and assume that the univariate marginal distribution functions $F_{d}$ are standard Fréchet, $F_{d}\left(x_{d}\right)=\exp \left\{-x_{d}^{-1}\right\}$ for $x_{d}>0, d=$ $1, \ldots, D$. Let $\tilde{\boldsymbol{Y}}=\left\{\tilde{\boldsymbol{Y}}_{i}=\left(\tilde{Y}_{i, 1}, \ldots, \tilde{Y}_{i, D}\right), i \in \mathbb{Z}\right\}$ be the associated $D$-variate sequence of i.i.d. random vectors with the same marginal distribution and let $\boldsymbol{M}_{n}=\left(\max _{i=1}^{n} Y_{i, 1}, \ldots, \max _{i=1}^{n} Y_{i, D}\right)$, and $\tilde{\boldsymbol{M}}_{n}$ similarly, denote the sequences of componentwise maxima. Then, for any $\boldsymbol{x}=\left(x_{1}, \ldots, x_{D}\right) \in \mathbb{R}_{+}^{D}$ and $[\mathbf{0}, \boldsymbol{x}]^{c}=$ $[\mathbf{0}, \infty] \backslash[\mathbf{0}, \boldsymbol{x}]$ we have by Theorem 3.1 in Samorodnitsky (2004), Proposition 2.1 of Smith and Weissman (1996) and a tightness argument that

$$
\begin{aligned}
& \lim _{n \rightarrow \infty} P\left(n^{-1} \boldsymbol{M}_{n} \leq \boldsymbol{x}\right)=\exp \left\{-\mu\left([\mathbf{0}, \boldsymbol{x}]^{c}\right)\right\}=G(\boldsymbol{x}), \\
& \lim _{n \rightarrow \infty} P\left(n^{-1} \tilde{\boldsymbol{M}}_{\boldsymbol{n}} \leq \boldsymbol{x}\right)=\exp \left\{-\tilde{\mu}\left([\mathbf{0}, \boldsymbol{x}]^{c}\right)\right\}=\tilde{G}(\boldsymbol{x})=P\left(\boldsymbol{Y}_{1} \leq \boldsymbol{x}\right),
\end{aligned}
$$

where $\mu(\cdot)$ and $\tilde{\mu}(\cdot)$ denote the exponent measures as in Resnick (1987). Then, for $\boldsymbol{v} \in[\mathbf{0}, \infty) \backslash\{\mathbf{0}\} \subseteq \mathbb{R}_{+}^{D}$, the function

$$
\theta(\boldsymbol{v})=\frac{\mu\left(\left[\mathbf{0}, \boldsymbol{v}^{-1}\right]^{c}\right)}{\tilde{\mu}\left(\left[\mathbf{0}, \boldsymbol{v}^{-1}\right]^{c}\right)},
$$

introduced by Nandagopalan (1994), is called the multivariate extremal index. For $D=1$ the quotient of the exponent measures reduces to the well-known univariate extremal index $\theta \in(0,1]$, cf. Leadbetter (1983). We will denote by $\theta_{d}$ the extremal index of the $d$-th sequence $\left\{Y_{i, d}, i \in \mathbb{Z}\right\}$. As mentioned above, $\theta(\boldsymbol{v})$ is the univariate extremal index of the series $\left\{\max _{d} v_{d} Y_{i, d}, i \in \mathbb{Z}\right\}$ (Smith and Weissman 1996, Proposition 2.1). 
In the following let $\boldsymbol{\theta}=\left(\theta_{1}, \ldots, \theta_{D}\right)$, and let $\boldsymbol{\theta} \boldsymbol{v}=\left(\theta_{1} v_{1}, \ldots, \theta_{D} v_{D}\right)$ denote the componentwise multiplication. Then,

$$
\theta(\boldsymbol{v})=\frac{l(\boldsymbol{\theta} \boldsymbol{v})}{\tilde{l}(\boldsymbol{v})},
$$

where $l$ and $\tilde{l}$ are the two stable tail dependence functions (Huang 1992),

$$
\begin{array}{ll}
l\left(z^{-1}\right)=\mu\left([\mathbf{0}, \boldsymbol{x}]^{c}\right), & z_{d}=-\left(\ln G_{d}\left(x_{d}\right)\right)^{-1}, d=1, \ldots, D, \\
\tilde{l}\left(\tilde{z}^{-1}\right)=\tilde{\mu}\left([\mathbf{0}, \boldsymbol{x}]^{c}\right), & \tilde{z}_{d}=-\left(\ln \tilde{G}_{d}\left(x_{d}\right)\right)^{-1}=x_{d}, d=1, \ldots, D .
\end{array}
$$

Up to now there are five known properties characterizing $\theta(\boldsymbol{v})$ (Beirlant et al. 2004; Nandagopalan 1994; Perfekt 1997; Smith and Weissman 1996):

$$
\begin{aligned}
& \theta(\boldsymbol{v}) \text { is continuous in } \boldsymbol{v}, \\
& \theta(c \boldsymbol{v})=\theta(\boldsymbol{v}), \text { for any constant } c>0, \\
& \theta\left(\boldsymbol{e}_{d}\right)=\theta_{d}, \text { where } \boldsymbol{e}_{d} \text { is the } d \text { th unit vector, } \\
& 0 \leq \theta(\boldsymbol{v}) \leq 1, \text { i.e. } l(\boldsymbol{\theta} \boldsymbol{v}) \leq \tilde{l}(\boldsymbol{v}), \\
& \theta_{d}>0 \text { for all } d=1, \ldots, D \text { iff } \theta(\boldsymbol{v})>0 \text { for all } \boldsymbol{v} \in[\mathbf{0}, \infty) \backslash\{\boldsymbol{0}\} .
\end{aligned}
$$

By property (T2) we may confine our analysis to the $(D-1)$-dimensional unit simplex $\mathbb{S}_{D}=\left\{\boldsymbol{v} \in[\mathbf{0}, \mathbf{1}]:\|\boldsymbol{v}\|_{1}=v_{1}+\ldots+v_{D}=1\right\}$ and refer to the restriction of $l$ and $\tilde{l}$ to $\mathbb{S}_{D}$ as (Pickands) dependence functions, cf. Pickands (1981) and Beirlant et al. (2004). We will frequently make use of the following properties (Beirlant et al. 2004):

$$
l_{\text {min }}(\boldsymbol{v})=\max \left\{v_{1}, \ldots, v_{D}\right\} \leq l(\boldsymbol{v}) \leq l_{\max }(\boldsymbol{v})=\sum_{d=1}^{D} v_{d},
$$

$$
l(v) \text { is convex, }
$$

$$
l(c \boldsymbol{v})=c l(\boldsymbol{v}), \text { for any constant } c>0,
$$

where $\boldsymbol{v} \in[\mathbf{0}, \infty) \backslash\{\boldsymbol{0}\}$, and $l_{\min }$ and $l_{\max }$ are valid dependence functions. Also, for later reference, let $A$ be a subset of $\{1, \ldots, D\}$ and let $\boldsymbol{e}_{A}$ be a vector in $\mathbb{R}^{D}$ with the $d$-th component equal to one if $d \in A$ and zero otherwise. Let $\mathbf{1}=\boldsymbol{e}_{\{1, \ldots, D\}}$.

Properties (T1) to (T5) above are not sufficient to characterize the function $\theta(v)$ completely. As a step towards a better understanding of the multivariate extremal index it is one of our main results to refine property (T4). In addition to Smith and Weissman's (1996) conjecture of $l(\boldsymbol{\theta} \boldsymbol{v}) \leq \tilde{l}(\boldsymbol{v})$ to be the only restriction on the two dependence functions we show further constraints in Section 3 which, equivalently, correspond to improved bounds for the function $\theta(\boldsymbol{v})$ given marginal dependence in terms of $\theta_{d}, d=1, \ldots, D$. In Section 4 the extremal coefficient, $\tilde{\phi}=\tilde{l}(\mathbf{1})$, a well-known summary measure for $\tilde{\mu}\left([\mathbf{0}, \boldsymbol{x}]^{c}\right)$, is related to the multivariate extremal index, cf. Smith (1990) and Schlather and Tawn (2003). We first discuss an obvious connection between the univariate extremal indices and the extremal coefficient and give an improved upper bound for the dependence adjusted extremal coefficient, $\phi=l(\boldsymbol{\theta 1})$, a counterpart of $\tilde{\phi}$ that applies to stationary sequences, see Martins and Ferreira (2005a). In the main, however, we will concentrate on the fact that $\theta(\mathbf{1})=\phi / \tilde{\phi}$. 
Now, knowledge of $\tilde{\phi}$ or $\phi$, respectively, allows for a significant improvement of the unrestricted bounds for $\theta(\boldsymbol{v})$ as given in Section 3. Throughout the text we will discuss various example processes.

\section{Properties of the multivariate extremal index}

A $D$-dimensional process $\boldsymbol{Y}=\left\{\boldsymbol{Y}_{i}=\left(Y_{i, 1}, \ldots, Y_{i, D}\right), i \in \mathbb{Z}\right\}$ is max-stable with Fréchet margins iff

$$
Y_{i, d}=\max _{j \geq 1} f_{i, d}\left(S_{j}\right) U_{j}, \quad i \in \mathbb{Z}, d=1, \ldots, D,
$$

where $\left\{U_{j}, S_{j}\right\}_{j=1}^{\infty}$ is a Poisson point process on $\mathbb{R}_{+} \times[0,1]$ with intensity $\mathrm{d} u / u^{2} \times \mathrm{d} s$, and $\left\{f_{i, d}\right\}_{i \in \mathbb{Z}}, d=1, \ldots, D$, are sequences of nonnegative deterministic spectral functions (de Haan and Pickands 1986). Replacing $\left\{f_{i}\right\} \sim$ $\left\{f_{i+1}\right\}$ by

$$
\left\{\left(\tilde{f}_{D(i-1)+1}, \ldots, \tilde{f}_{D i}\right)\right\} \sim\left\{\left(\tilde{f}_{D(i-1+k)+1}, \ldots, \tilde{f}_{D(i+k)}\right)\right\}
$$

in Theorem 5.1 of de Haan and Pickands (1986) with $\tilde{f}_{D(i-1)+d}:=f_{i, d}$ gives the spectral representation for stationary $D$-variate max-stable processes.

Theorem 1 The elements of a stationary max-stable D-variate process $\boldsymbol{Y}$ are representable by Eq. 2 with proper sequence $\left\{f_{i}=\left(f_{i, 1}, \ldots, f_{i, D}\right)\right\}_{i \in \mathbb{Z}}$. There exists a piston $\Gamma$ such that $f_{i+1} \equiv \Gamma\left(f_{i}\right)$.

Let $[0,1]=S_{1} \cup S_{2}$ be the Hopf decomposition for Eq. 2 into the dissipative and the conservative part (Krengel 1985), where $S_{1}$ is isomorphic to $S_{0} \times \mathbb{Z}$ for some measurable set $S_{0} \subset S_{1}$. Theorem 3.1 in Samorodnitsky (2004) states that the extremal index $\theta_{d}$ of the $d$ th component is given by

$$
\theta_{d}=\int_{S_{0}} \max _{i \in \mathbb{Z}} f_{i, d}(s) \mathrm{d} s .
$$

By means of Proposition 2.1 in Smith and Weissman (1996) we may conclude that the multivariate extremal index equals

$$
\theta(\boldsymbol{v})=\frac{\int_{S_{0}} \max _{i \in \mathbb{Z}} \max _{d=1}^{D} v_{d} f_{i, d}(s) \mathrm{d} s}{\int_{S_{0}} \sum_{i \in \mathbb{Z}} \max _{d=1}^{D} v_{d} f_{i, d}(s) \mathrm{d} s+\int_{S_{2}} \max _{d=1}^{D} v_{d} f_{0, d}(s) \mathrm{d} s}, \quad \boldsymbol{v} \in \mathbb{S}_{D} .
$$

Theorem 2 The set of extremal index functions is closed under uniform convergence.

Proof We show that for any sequence $\theta_{m}$ there exists an extremal index function $\theta$ and a subsequence $\theta_{m_{k}}$ with $\theta_{m_{k}} \rightarrow \theta$ uniformly. Assume that the 
$\theta_{m}$ are the extremal index functions of some processes with spectral functions $f_{m, i}(s)=\left(f_{m, i, 1}(s), \ldots, f_{m, i, D}(s)\right), s \in[0,1]$. Choose suitable measurable functions $i_{m, k}(s), k=1, \ldots, D$, so that for all $s \in S_{1}$ and $\boldsymbol{v} \in \mathbb{S}_{D}$ we have $\max _{i} \max _{d=1}^{D} v_{d} f_{m, i, d}(s)=\max _{k=1}^{D} \max _{d=1}^{D} v_{d} f_{m, i_{m, k}(s), d}(s)$, and the $h_{m, i}$ with

$$
h_{m, i}(s)= \begin{cases}0, & i=i_{m, k}(s), k=1, \ldots, D, s \in S_{1} \\ f_{m, i}(s), & \text { otherwise }\end{cases}
$$

are the spectral functions of a $D$-variate stationary max-stable process given by Eq. 2 .

Denote by $H_{m}$ its $D$-variate marginal distribution and let $g_{m}(s)=$ $\left(f_{m, i_{m, 1}(s)}(s), \ldots, f_{m, i_{m, D}(s)}(s)\right) \in \mathbb{R}^{D \times D}$ for $s \in S_{1}$ and 0 otherwise. Then

$$
G_{m}(A)=\int_{\left\{s: g_{m}(s) /\left\|g_{m}(s)\right\|_{1} \in A\right\}}\left\|g_{m}(s)\right\|_{1} \mathrm{~d} s, \quad A \in B,
$$

defines a measure $G_{m}$ on the Borel sets $B$ of $\mathbb{S}_{D^{2}}$.

Let the Poisson point processes $\left\{U_{j}, N_{j}, \eta_{j}\right\}_{j=1}^{\infty}$ on $\mathbb{R}_{+} \times \mathbb{Z} \times \mathbb{R}^{D \times D}$ with intensity $\mathrm{d} u / u^{2} \times 1 \times \mathrm{d} G_{m}$ and $\left\{V_{j}, \xi_{j}\right\}_{j=1}^{\infty}$ on $\mathbb{R}_{+} \times \mathbb{R}^{D}$ with intensity $\mathrm{d} u / u^{2} \times$ $\mathrm{d} H_{m}$ be independent. Define

$$
Y_{m, i, d}=\max \left\{\max _{j \geq 1} \zeta_{j, i-N_{j}, d} U_{j}, \max _{j \geq 1} \xi_{j, d} V_{j}\right\}
$$

where $\eta_{j}=\left(\eta_{j, 1,1}, \ldots, \eta_{j, 1, D}, \eta_{j, 2,1}, \ldots, \eta_{j, D, D}\right)$ and $\zeta_{j, i, d}=\eta_{j, i, d}$, for $i, d=$ $1, \ldots, D$, and 0 otherwise. Then, we have for the extremal index function $\theta_{Y_{m}}$ of $Y_{m}$ :

$\theta_{Y_{m}}(\boldsymbol{v})=\frac{\int \max _{k=1}^{D} \max _{d} a_{k, d} v_{d} G_{m}(\mathrm{~d} a)}{\int \sum_{k=1}^{D} \max _{d} a_{k, d} v_{d} G_{m}(\mathrm{~d} a)+\int \max _{d} b_{d} v_{d} H_{m}(\mathrm{~d} b)}=\theta_{m}(\boldsymbol{v}), \quad \boldsymbol{v} \in \mathbb{S}_{D}$,

where $a=\left(a_{1,1}, \ldots, a_{1, D}, a_{2,1}, \ldots, a_{D, D}\right)$ and $b=\left(b_{1}, \ldots, b_{D}\right)$.

There exists a subsequence $\left(m_{k}\right)$ such that $G_{m_{k}} \rightarrow G$ on $\mathbb{S}_{D^{2}}$ and $H_{m_{k}} \rightarrow H$ on $\mathbb{S}_{D}$ for some finite measures $G$ and $H$. Define a max-stable process $Y$ by means of $G, H$ and Eq. 3. Let $\theta$ be its multivariate extremal index function. Then, the convergence of $\theta_{m_{k}} \rightarrow \theta$ is uniform, since the denominator in Eq. 4 is uniformly bounded away from 0 and the three integrands in Eq. 4 are bounded and uniformly continuous in $v \in \mathbb{S}_{D}, b \in \mathbb{S}_{D}, a \in \mathbb{S}_{D^{2}}$.

In the remainder we shall follow the ideas of Deheuvels (1983) and Smith and Weissman (1996) who consider so-called $R S$ processes which are discrete and stationary versions of Eq. 2 given by

$$
Y_{i d}=\max \left\{R_{i d}, S_{i d}\right\}
$$

where

$$
R_{i d}=\max _{j \in I} \max _{k \in \mathbb{Z}} a_{j k d} Z_{j, i-k} \quad \text { and } \quad S_{i d}=\max _{j \in F} \max _{0 \leq k \leq N_{j}} \alpha_{j k d} Z_{j, i-k}^{*}
$$


Here, $I, F \subseteq \mathbb{N} \cup\{0\},\left\{Z_{j, i}, i \in \mathbb{Z}, j \in I\right\}$ and $\left\{Z_{j, i}^{*}, 0 \leq i \leq N_{j}<\infty, j \in F\right\}$ are independent sequences of i.i.d. standard Fréchet variables, and $Z_{j, i}^{*}=$ $Z_{j, i+m\left(N_{j+1}\right)}^{*}, m \in \mathbb{Z}$. The constants $a_{j k d}, j \in I, k \in \mathbb{Z}$, and $\alpha_{j k d}, j \in F, 0 \leq k \leq$ $N_{j}$ are non-negative with $\sum_{j} \sum_{k} a_{j k d}+\sum_{j} \sum_{k} \alpha_{j k d}=1$ for $d=1, \ldots, D$. Note that the $S_{i d}$ part of the $R S$ process (Eq. 5) consists of periodic elements and leads to non-ergodic processes (Stoev 2007), whereas the mixing component $R_{i d}$ corresponds to the $M_{4}$ class discussed by Smith and Weissman (1996). For the dependence functions of an $R S$ process we have, cf. Eq. 1,

$$
\begin{aligned}
l(\boldsymbol{\theta} \boldsymbol{v}) & =\sum_{j \in I} \max _{k \in \mathbb{Z}} \max _{d=1, \ldots, D} a_{j k d} v_{d}, \\
\tilde{l}(\boldsymbol{v}) & =\sum_{j \in I} \sum_{k \in \mathbb{Z}} \max _{d=1, \ldots, D} a_{j k d} v_{d}+\sum_{j \in F} \sum_{0 \leq k \leq N_{j}} \max _{d=1, \ldots, D} \alpha_{j k d} v_{d} .
\end{aligned}
$$

The following proposition allows to generalize results upon the multivariate extremal index from $M_{4}$ processes to stationary max-stable processes. Under the conditions of Theorem 2.3 of Smith and Weissman (1996) the results obtained in Sections 3 and 4 hold true also for general stationary processes in the maximum domain of attraction of a max-stable process.

Proposition 1 The multivariate extremal index of a D-variate stationary maxstable process $\boldsymbol{Y}$ may be approximated uniformly by the multivariate extremal index of an $M_{4}$ process.

Proof For a given multivariate extremal index $\theta$ there exists a corresponding process $Y_{0}$ with standard Fréchet margins of the form (3) with measures $G$ and $H$. Let $\varepsilon>0$ and consider discrete versions $G_{1}$ and $H_{1}$ of $G$ and $H$, respectively, such that the corresponding process $Y_{1}$ has standard Fréchet margins and the extremal index $\theta_{Y_{1}}$ satisfies $\left|\theta(\boldsymbol{v})-\theta_{Y_{1}}(\boldsymbol{v})\right|<\varepsilon / 2$ for all $\boldsymbol{v} \in \mathbb{S}_{d}$. Let $2 D / \varepsilon<L \in \mathbb{N}$ and let the Poisson point processes $\left\{U_{j}, N_{j}, \eta_{j}\right\}_{j=1}^{\infty}$ on $\mathbb{R}_{+} \times$ $\mathbb{Z} \times \mathbb{R}^{D \times D}$ with intensity $\mathrm{d} u / u^{2} \times 1 \times \mathrm{d} G_{1}$ and $\left\{V_{j}, M_{j}, \xi_{j}\right\}_{j=1}^{\infty}$ on $\mathbb{R}_{+} \times \mathbb{Z} \times \mathbb{R}^{D}$ with intensity $\mathrm{d} u / u^{2} \times 1 \times \mathrm{d} H_{1} / L$ be independent. Define $Y_{2}$ by

$$
Y_{2, i, d}=\max \left\{\max _{j} \zeta_{j, i-N_{j}, d} U_{j}, \max _{j} v_{j, i-M_{j}, d} V_{j}\right\}
$$

where $\zeta_{j, i, d}=\eta_{j, i, d}$, for $i=1, \ldots, D$, and 0 otherwise, and $v_{j, i, d}=\xi_{j, i, d}$, for $i=$ $1, \ldots, L$, and 0 otherwise. Then, $Y_{2}$ is an $M_{4}$-process and its extremal index function $\theta_{Y_{2}}$ satisfies for all $\boldsymbol{v} \in \mathbb{S}_{D}$ :

$$
\left|\theta_{Y_{2}}(\boldsymbol{v})-\theta(\boldsymbol{v})\right|<\left|\theta_{Y_{2}}(\boldsymbol{v})-\theta_{Y_{1}}(\boldsymbol{v})\right|+\left|\theta_{Y_{1}}(\boldsymbol{v})-\theta(\boldsymbol{v})\right|<D / L+\varepsilon / 2<\varepsilon .
$$

Whenever we will define in the remainder of the paper an $R S$ or $M_{4}$ process by its coefficients in Eq. 5 we will tacitly assume that all coefficients not explicitly defined be zero. 


\section{Bounds for the multivariate extremal index}

We now turn to the question of the interdependencies between $l$ and $\tilde{l}$. From the definition of the multivariate extremal index it merely follows that $l(\theta v) \leq$ $\tilde{l}(\boldsymbol{v})$, cf. Smith and Weissman's (1996) conjecture. We give the following counterexample to demonstrate that $l(\boldsymbol{\theta} v) \leq \tilde{l}(\boldsymbol{v})$ is not a sufficient condition for $l(\boldsymbol{\theta} v) / \tilde{l}(\boldsymbol{v})$ to serve as a valid multivariate extremal index.

Example 1 Consider an $M_{4}$ process with $D=2, I=2$ and $\theta_{1}=\theta_{2}=0.5$. The condition $l(\boldsymbol{\theta v}) / \tilde{l}(\boldsymbol{v}) \leq 1$ allows for $\tilde{\phi}=\tilde{l}(\mathbf{1})=\sum_{j} \sum_{k} \max _{d} a_{j k d}=1$, say, which is equivalent to $a_{j k 1}=a_{j k 2}$ for all $j, k$, see also Corollary 2 below. Then, it necessarily follows that $l(\boldsymbol{\theta} \boldsymbol{v})=\sum_{j} \max _{k} \max _{d} a_{j k d} v_{d}=0.5 \max _{d} v_{d}$ obviously further restricting the requirement $l(\boldsymbol{\theta} \boldsymbol{v}) / \tilde{l}(\boldsymbol{v}) \leq 1$. Accordingly, $\theta_{1}=\theta_{2}=0.5$ is incompatible with $\theta(\mathbf{1})=1$, for example. See Fig. 1 for a sketch of the valid bounds of $\theta(\mathbf{1})$ as derived from the results below.

For an in-depth illustration of the reasoning in Example 1 we introduce a decomposition for $\tilde{l}$ in the subsequent theorem and state simple but im-

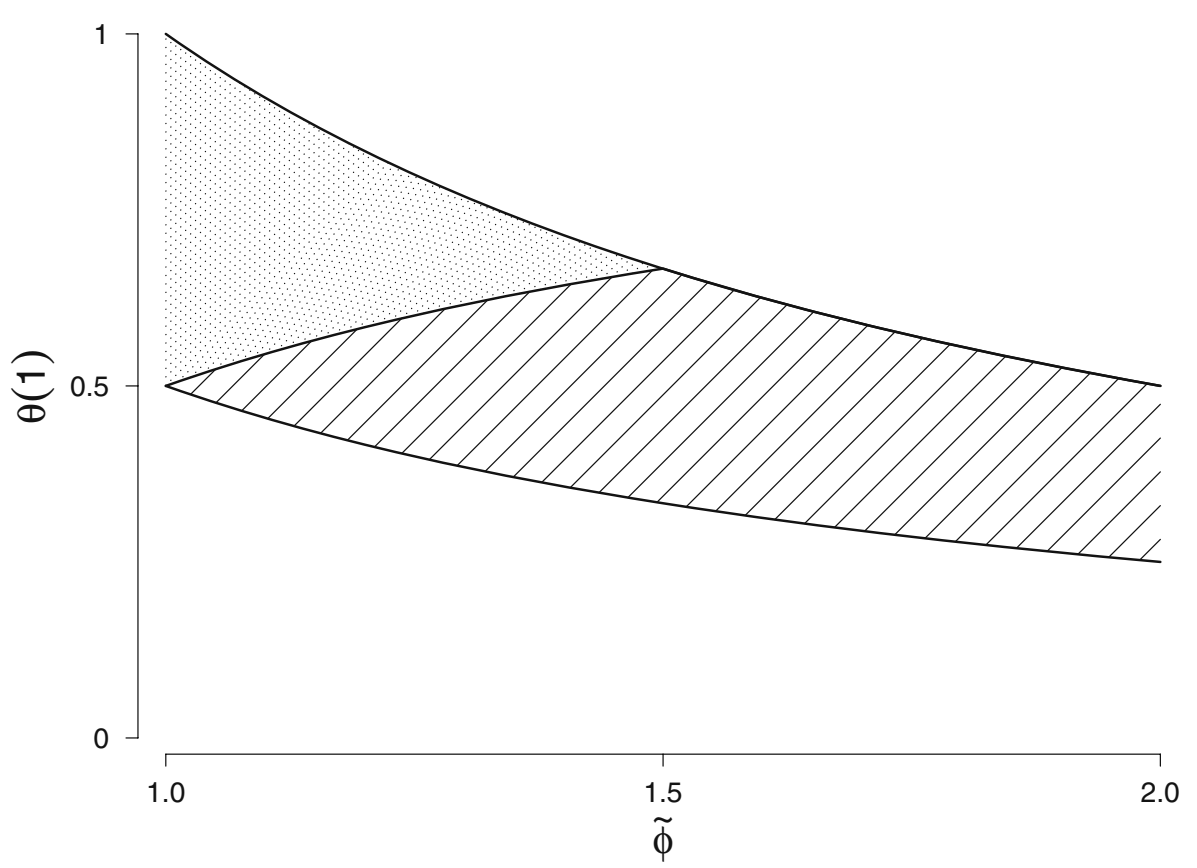

Fig. 1 Bounds for $\theta(\mathbf{1})$ with $\theta_{1}=\theta_{2}=0.5$ as functions of the extremal coefficient, see Example 1. Lined: Valid combinations. Gray: Invalid combinations, but consistent with the bound $l(\boldsymbol{\theta} \boldsymbol{v}) \leq \tilde{l}(\boldsymbol{v})$ given by Smith and Weissman (1996). 
portant properties of which we shall repeatedly make use in the rest of the paper.

Lemma 1 Let $\widehat{\sum}_{k} a_{k}=\sum_{k} a_{k}-\max _{k} a_{k}$ for any sequence of nonnegative constants $a_{k}$ and assume that $\max _{k} a_{k}$ exists. Then, for any $a_{k d} \in \mathbb{R}$

$$
\max _{d} \widehat{\sum_{k}} a_{k d} \leq \widehat{\sum_{k}} \max _{d} a_{k d}
$$

Proof For any fixed $d^{*} \in\{1, \ldots, D\}$ let $\varepsilon=\sum_{k}\left\{\max _{d} a_{k d}-a_{k d^{*}}\right\}$. Now, $\varepsilon \geq$ $\max _{k} \max _{d} a_{k d}-\max _{k} a_{k d^{*}}$, and

$$
\sum_{k} a_{k d^{*}}+\max _{k} \max _{d} a_{k d}-\max _{k} a_{k d^{*}} \leq \sum_{k} \max _{d} a_{k d}, \quad \text { for all } d^{*} \in\{1, \ldots, D\} .
$$

Theorem 3 Let an arbitrary $R S$ process with extremal indices $\theta_{1}, \ldots, \theta_{D}$ be given by the coefficients $a_{j k d}, j \in I, k \in \mathbb{Z}, d=1, \ldots, D$, and $\alpha_{j k d}, j \in F, 0 \leq$ $k \leq N_{j}, d=1, \ldots, D$. For all $j, d$ let $a_{j k d}^{*}=0$ for all $k$ except one $k=k(j, d) \in$ $\arg \max _{k} a_{j k d}$ where $a_{j k d}^{*}=\max _{k} a_{j k d}$, and $\hat{a}_{j k d}=0$ for $k=k(j, d)$ and $\hat{a}_{j k d}=$ $a_{j k d}$ otherwise, i.e. $\hat{a}_{j k d}=a_{j k d}-a_{j k d}^{*}$. Define $\tilde{l}_{\theta}(\boldsymbol{v})=\sum_{j} \sum_{k} \max _{d} a_{j k d}^{*} v_{d}$ and $\tilde{l}_{1-\theta}(\boldsymbol{v})=\sum_{j} \sum_{k} \max _{d} \hat{a}_{j k d} v_{d}+\sum_{j} \sum_{k} \max _{d} \alpha_{j k d}$.

(1) The functions $\tilde{l}_{\theta}$ and $\tilde{l}_{1-\theta}$ are valid dependence functions with sharp upper and lower bounds given by $\tilde{l}_{\theta, \min }(\boldsymbol{v})=l_{\min }(\boldsymbol{\theta} v)=\max _{d} \theta_{d} v_{d}$, $\tilde{l}_{\theta, \max }(\boldsymbol{v})=l_{\max }(\boldsymbol{\theta} \boldsymbol{v})=\sum_{d} \theta_{d} v_{d}, \tilde{l}_{1-\theta, \min }(\boldsymbol{v})=\max _{d}\left(1-\theta_{d}\right) v_{d}$, and $\tilde{l}_{1-\theta, \max }(\boldsymbol{v})=$ (2) It holds that

$$
\begin{gathered}
\tilde{l}_{\theta, \min }(\boldsymbol{v}) \leq l(\boldsymbol{\theta} \boldsymbol{v}) \leq \tilde{l}_{\theta}(\boldsymbol{v}), \\
l(\boldsymbol{\theta v})+\tilde{l}_{1-\theta, \min }(\boldsymbol{v}) \leq \tilde{l}(\boldsymbol{v}) \leq \tilde{l}_{\theta}(\boldsymbol{v})+\tilde{l}_{1-\theta}(\boldsymbol{v}),
\end{gathered}
$$

where equality applies for the last inequality iff for all $j \in I$ and $d=$ $1, \ldots, D$ we have $\hat{a}_{j k d}=0$ for all $k \in\{k(j, 1), \ldots, k(j, D)\}$.

(3) For any function $l(\boldsymbol{\theta} v)$ a corresponding $M_{4}$ process exists such that $\tilde{l}(\boldsymbol{v})=1, \boldsymbol{v} \in \mathbb{S}_{D}$.

Proof

(1) From $\tilde{l}_{\theta}\left(\boldsymbol{\theta}^{-1} \boldsymbol{v}\right)=\sum_{j} \sum_{k} \max _{d} a_{j k d}^{*} v_{d} / \theta_{d}$ it follows that the function $\tilde{l}_{\theta}$ is a dependence function. Analogously for $\tilde{l}_{1-\theta}$. Now, the assertion follows from condition (L1). 
(2) Ineq. 6 follows directly from (1) and the respective definitions. Concerning the left hand side of Eq. 7, we get by (1) and Lemma 1 that for all $\boldsymbol{v} \in \mathbb{S}_{D}$

$$
\begin{aligned}
l(\boldsymbol{\theta} \boldsymbol{v}) & +\tilde{l}_{1-\theta, \min }(\boldsymbol{v}) \\
& =l(\boldsymbol{\theta} \boldsymbol{v})+\max _{d}\left\{\sum_{j} \sum_{k} \alpha_{j k d} v_{d}+\sum_{j} \sum_{k} a_{j k d} v_{d}-\sum_{j} \max _{k} a_{j k d} v_{d}\right\} \\
& \leq l(\boldsymbol{\theta} \boldsymbol{v})+\max _{d}\left\{\sum_{j} \sum_{k} \alpha_{j k d} v_{d}\right\}+\max _{d}\left\{\sum_{j} \widehat{\sum_{k}} a_{j k d} v_{d}\right\} \\
& \leq l(\boldsymbol{\theta} \boldsymbol{v})+\sum_{j} \sum_{k} \max _{d} \alpha_{j k d} v_{d}+\sum_{j} \sum_{k} \max _{d} a_{j k d} v_{d}-\sum_{j} \max _{k} \max _{d} a_{j k d} v_{d} \\
& =\sum_{j} \sum_{k} \max _{d} a_{j k d} v_{d}+\sum_{j} \sum_{k} \max _{d} \alpha_{j k d} v_{d}=\tilde{l}(\boldsymbol{v}) .
\end{aligned}
$$

Finally, the right hand side of Eq. 7 follows from the fact that $\max _{d} a_{j k d}^{*} v_{d}+\max _{d} \hat{a}_{j k d} v_{d} \geq \max _{d}\left(a_{j k d}^{*}+\hat{a}_{j k d}\right) v_{d}$. Equality holds for all $\boldsymbol{v} \in \mathbb{S}_{D}$ iff $\hat{a}_{j k d}=0$ for all $k \in\{k(j, 1), \ldots, k(j, D)\}$.

(3) Note that the swapping of the values of $a_{j k_{1} d}$ and $a_{j k_{2} d}$ does not change $l$ for any $j, k_{1}, k_{2}, d$, so that we may assume that for all $j$ and $k a_{j k d}^{*} \neq 0$ for at most one value of $d$. Then,

$$
\tilde{l}_{\theta}(\boldsymbol{v})=\sum_{j} \sum_{k} \max _{d} a_{j k d}^{*} v_{d}=\sum_{j} \sum_{d} a_{j, k(j, d), d}^{*} v_{d}=\sum_{d} \theta_{d} v_{d} .
$$

Further, for all $j$ and $k$, let the $\hat{a}_{j k d}$ be such that $\hat{a}_{j k d} \neq 0$ for at most one value of $d$, and $\hat{a}_{j k d}=0$ for all $j, d$ and $k \in\{k(j, 1), \ldots, k(j, D)\}$. Then, $\tilde{l}_{1-\theta}(\boldsymbol{v})=\sum_{d}\left(1-\theta_{d}\right) v_{d}$ by the above argumentation. Finally, by (2) we have $\tilde{l}(\boldsymbol{v})=\tilde{l}_{\theta}(\boldsymbol{v})+\tilde{l}_{1-\theta}(\boldsymbol{v})=1, \boldsymbol{v} \in \mathbb{S}_{D}$.

Now, the incompatibility of $\theta_{1}=\theta_{2}=0.5$ and $\theta(\mathbf{1})=1$, i.e. $l(\boldsymbol{\theta} \mathbf{1})=\tilde{l}(\mathbf{1})$, in Example 1 is highlighted additionally by Ineq. 7. There, we find that $\theta_{d}=1, d=$ $1, \ldots, D$, or equivalently, $\tilde{l}_{1-\theta, \min }(\mathbf{1})=0$ is a necessary condition for $\theta(\mathbf{1})=1$. In addition to Theorem 3 (1) and (2) see also Smith and Weissman (1996) who discuss a special case for which $\tilde{l}(\boldsymbol{v})-l(\boldsymbol{\theta} \boldsymbol{v})$ is convex. There, using the notation of Theorem 3, for $D=2$ a process with $k(j, 1)=k(j, 2), j \in I$ is considered. Then, by Theorem 3 (2) we have that $\tilde{l}(\boldsymbol{v})=\tilde{l}_{\theta}(\boldsymbol{v})+\tilde{l}_{1-\theta}(\boldsymbol{v})=l(\boldsymbol{\theta v})+\tilde{l}_{1-\theta}(\boldsymbol{v})$. Now, $\tilde{l}(\boldsymbol{v})-l(\boldsymbol{\theta} v)$ is a valid dependence function by Theorem 3 (1) and hence also a convex function. The fact that, in general, $\tilde{l}(\boldsymbol{v})-l(\boldsymbol{\theta} \boldsymbol{v})$ may be neither a dependence function nor a convex function at all does not, however, allow for the conclusion of arbitrariness of $l$ and $\tilde{l}$ as will become clear in the rest of the paper. 
Theorem 4 below gives sharp upper and lower bounds for $\theta(\boldsymbol{v})=l(\boldsymbol{\theta} \boldsymbol{v}) / \tilde{l}(\boldsymbol{v})$ for all $\boldsymbol{v} \in \mathbb{S}_{D}$ given $\theta_{d}, d=1, \ldots, D$, i.e. bounds for the entire multivariate extremal index function given only marginal dependence in terms of $\theta\left(\boldsymbol{e}_{d}\right)$, $d=1, \ldots, D$.

Theorem 4 Let $\Theta\left(\theta_{1}, \ldots, \theta_{D}\right)$ be the closed set of multivariate extremal index functions of all stationary max-stable processes with univariate extremal indices $\theta_{d} \in(0,1], d=1, \ldots, D$. Define

$$
\begin{aligned}
\theta_{\text {inf }}: \mathbb{S}_{D} \rightarrow(0,1], \boldsymbol{v} \mapsto \inf _{\theta \in \Theta\left(\theta_{1}, \ldots, \theta_{D}\right)} \theta(\boldsymbol{v}), \\
\theta_{\text {sup }}: \mathbb{S}_{D} \rightarrow(0,1], \boldsymbol{v} \mapsto \sup _{\theta \in \Theta\left(\theta_{1}, \ldots, \theta_{D}\right)} \theta(\boldsymbol{v}) .
\end{aligned}
$$

Then,

$$
\begin{aligned}
\theta_{\text {inf }}(\boldsymbol{v}) & =\max _{d} \theta_{d} v_{d}, \\
\theta_{\text {sup }}(\boldsymbol{v}) & =\frac{\sum_{d} \theta_{d} v_{d}}{\sum_{d} \theta_{d} v_{d}+\max _{d}\left(1-\theta_{d}\right) v_{d}} .
\end{aligned}
$$

In particular, $\theta_{\text {inf }}, \theta_{\text {sup }} \in \Theta\left(\theta_{1}, \ldots, \theta_{D}\right)$.

Proof Let $\mathcal{A}=\mathcal{A}\left(\theta_{1}, \ldots, \theta_{D}\right)$ be the class of $R S$ processes $A$ with coefficients $a_{j k d}, j \in I, k \in \mathbb{Z}, \alpha_{j k d}, j \in F, 0 \leq k \leq N_{j}$, such that $l\left(\boldsymbol{\theta} \boldsymbol{e}_{d}\right)=\theta_{d}, d=$ $1, \ldots, D$. Now, using the same notation as in Theorem 3 ,

$$
l_{\min }(\boldsymbol{\theta} \boldsymbol{v}) \leq \theta(\boldsymbol{v} \mid A) \leq \frac{l(\boldsymbol{\theta} \boldsymbol{v} \mid A)}{l(\boldsymbol{\theta} \boldsymbol{v} \mid A)+\tilde{l}_{1-\theta, \min }(\boldsymbol{v})} \leq \frac{l_{\max }(\boldsymbol{\theta v})}{l_{\max }(\boldsymbol{\theta} \boldsymbol{v})+\tilde{l}_{1-\theta, \min }(\boldsymbol{v})},
$$

where the lower bound is sharp by property (L1) and Theorem 3 (3), the second inequality holds with Theorem 3 (2) and the right hand side follows from the discussion of the mapping $x \mapsto \frac{x}{x+a}, x, a \geq 0$. To show that the upper bound is sharp consider $A^{*} \in \mathcal{A}$ with $I=\{1, \ldots, D\}, F=\{1\}, a_{d 1 d}^{*}=\theta_{d}$ and $\alpha_{11 d}^{*}=1-\theta_{d}$. Proposition 1 finalizes the proof.

Figure 2 gives a bivariate representation of the above bounds. Theorem 4 may equivalently be rewritten in terms of an improved lower bound for $\tilde{l}(\boldsymbol{v})$ making use of the additional information obtained by $l(\theta v)$, and an improved upper bound for $l(\boldsymbol{\theta} \boldsymbol{v})$ given $\theta_{d}$ and $\tilde{l}(\boldsymbol{v})$.

Corollary 1 For any stationary max-stable process with univariate extremal indices $\theta_{d} \in(0,1], d=1, \ldots, D$, it holds that for all $\boldsymbol{v} \in \mathbb{S}_{D}$

$$
l(\boldsymbol{\theta} \boldsymbol{v}) \frac{\sum_{d} \theta_{d} v_{d}+\max _{d}\left(1-\theta_{d}\right) v_{d}}{\sum_{d} \theta_{d} v_{d}} \leq \tilde{l}(\boldsymbol{v}) \leq 1,
$$




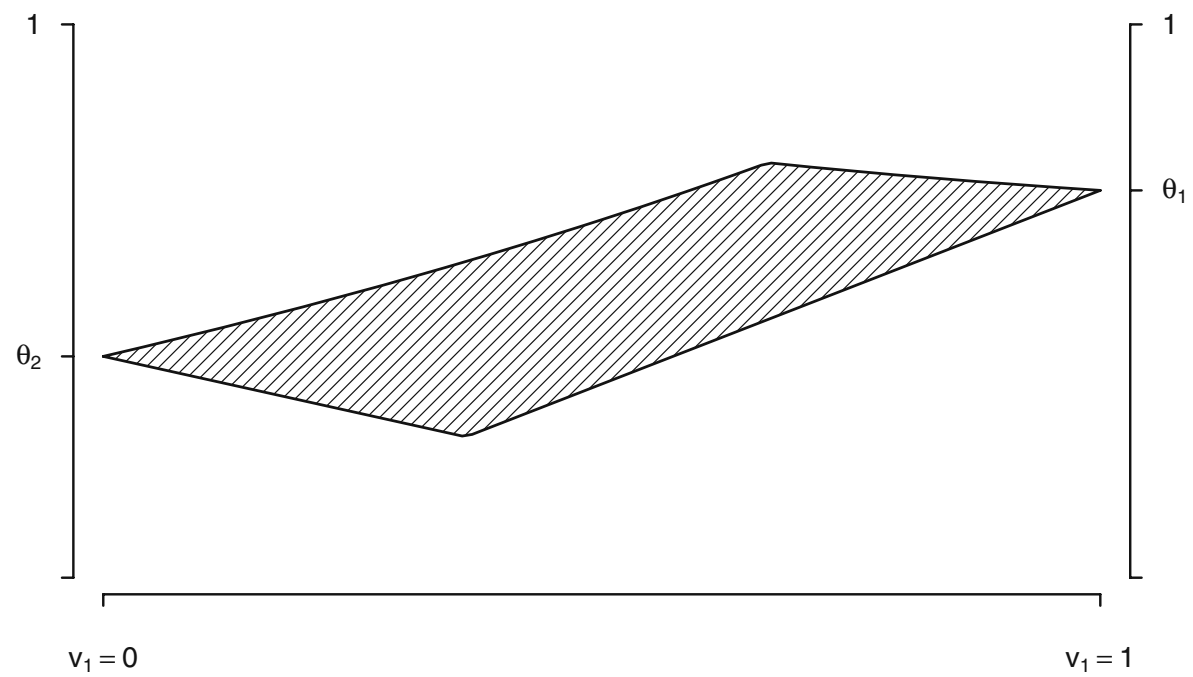

Fig. 2 Bounds for $\theta(\boldsymbol{v})$ in the bivariate case: All admissible multivariate extremal index functions for $\theta_{1}=0.7, \theta_{2}=0.4$ are located in the shaded area (including the boundaries).

and

$$
\max _{d} \theta_{d} v_{d} \leq l(\boldsymbol{\theta} \boldsymbol{v}) \leq \min \left\{\frac{\tilde{l}(\boldsymbol{v}) \sum_{d} \theta_{d} v_{d}}{\sum_{d} \theta_{d} v_{d}+\max _{d}\left(1-\theta_{d}\right) v_{d}}, \sum_{d} \theta_{d} v_{d}\right\} .
$$

\section{Exploring the extremal coefficients}

The extremal coefficient $\tilde{\phi}$ has been proposed as a summary measure for the in general complex dependence structure of $\tilde{G}(\boldsymbol{x})$ given by $\tilde{\mu}\left([\mathbf{0}, \boldsymbol{x}]^{c}\right)$, see Smith (1990). In effect, it is nothing but a single point of the respective dependence function, namely

$$
\tilde{\phi}=\tilde{l}(\mathbf{1})=D \tilde{l}\left(D^{-1} \mathbf{1}\right) .
$$

Nevertheless, due to properties (L1) and (L2) the extremal coefficient substantially restricts the possible shape of the entire dependence function $\tilde{l}$. Also, the extremal coefficient $\tilde{\phi}$ has been interpreted as the number of independent variables in a multivariate setting. For a discussion of its further properties see e.g. Schlather and Tawn (2003). So far, however, the bounds for $\theta(\boldsymbol{v})$ derived in Section 3 do not incorporate any information in terms of the extremal coefficient. Being a quotient measure of two dependence functions it is therefore natural with respect to $\theta(\boldsymbol{v})$ to consider the effect of a fixed extremal coefficient on the above bounds. Depending on the value of the extremal coefficient it turns out that the bounds may be improved significantly; 
compare Fig. 3 with Fig. 2. Following Martins and Ferreira (2005a) we may also look at

$$
\phi=l(\boldsymbol{\theta 1})=D l\left(D^{-1} \boldsymbol{\theta 1}\right)
$$

as a temporal dependence adjusted extremal coefficient. Here, a single point of the dependence function of $G(\boldsymbol{x})$ is held fixed thereby partially characterizing the entire function $l$ in the above sense. We therefore also discuss the corresponding improvement of the bounds for $\theta(v)$ given $\phi$, cf. Fig. 4. Note that the structure of the improvement of the bounds is completely distinct for fixed $\phi$ and $\tilde{\phi}$, respectively, being reflected by the differing complexity of the particular theorems below. Before we turn to the interrelationship between $\theta(\boldsymbol{v})$ and the two extremal coefficients we discuss how the latter themselves are influenced by marginal dependence.

Theorem 5 Let $A$ be a non-empty subset of $\{1, \ldots, D\}$. Then, for any stationary max-stable process with univariate extremal indices $\theta_{d} \in(0,1], d \in A$, the extremal coefficient $\tilde{\phi}_{A}$ is limited by the sharp bounds

$$
\max _{d \in A} \theta_{d}+\max _{d \in A}\left(1-\theta_{d}\right) \leq \tilde{\phi}_{A} \leq|A| .
$$

Proof The left inequality follows immediately from Theorem 3 (2). The right inequality is well-known, and sharp by Theorem 3 (3). An $M_{4}$ process with $I=\{1\}$ that reaches the lower bound is given by $a_{11 d}=\theta_{d}, a_{1 k d}=\left(1-\theta_{d}\right) / K$ for $k=2, \ldots, K+1$ and $K$ large enough such that $\theta_{d} \geq\left(1-\theta_{d}\right) / K$ for all $d$.

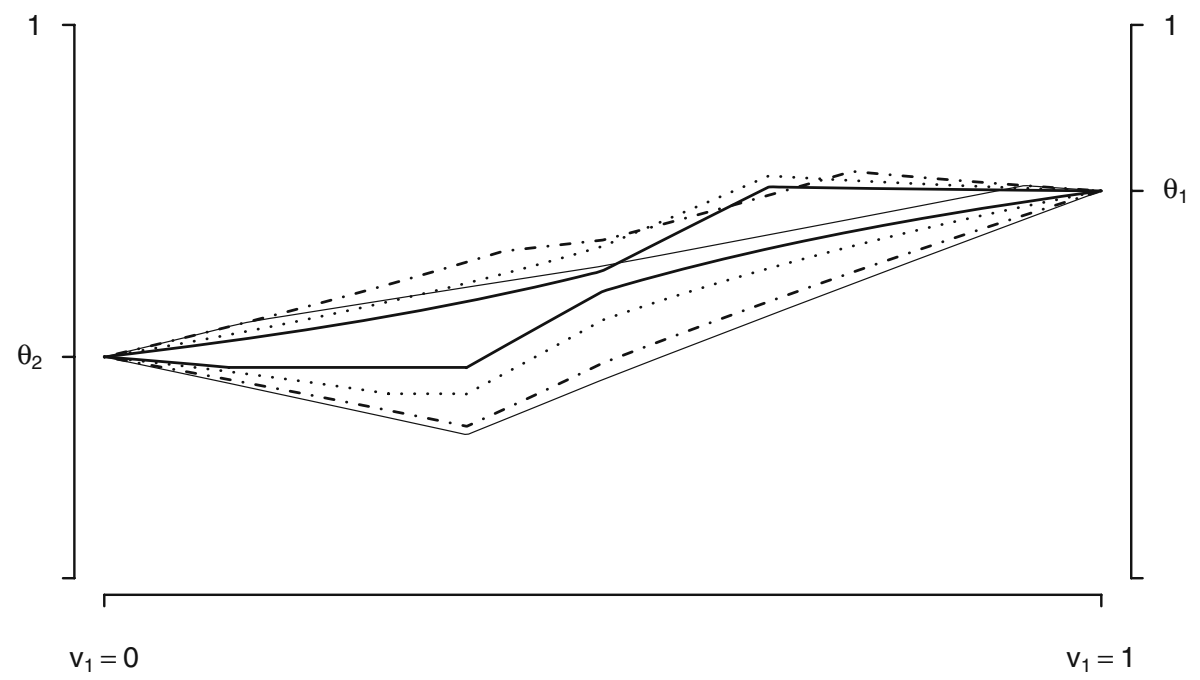

Fig. 3 Upper and lower bounds for $\theta(\boldsymbol{v})$ as in Theorems 7 and 8 given $\tilde{\phi}=1.35$ (thick line), $\tilde{\phi}=1.5$ (dotted line), $\tilde{\phi}=1.8$ (dash-dotted line) and $\tilde{\phi}=1.95$ (thin line) for $\theta_{1}=0.7, \theta_{2}=0.4$. 


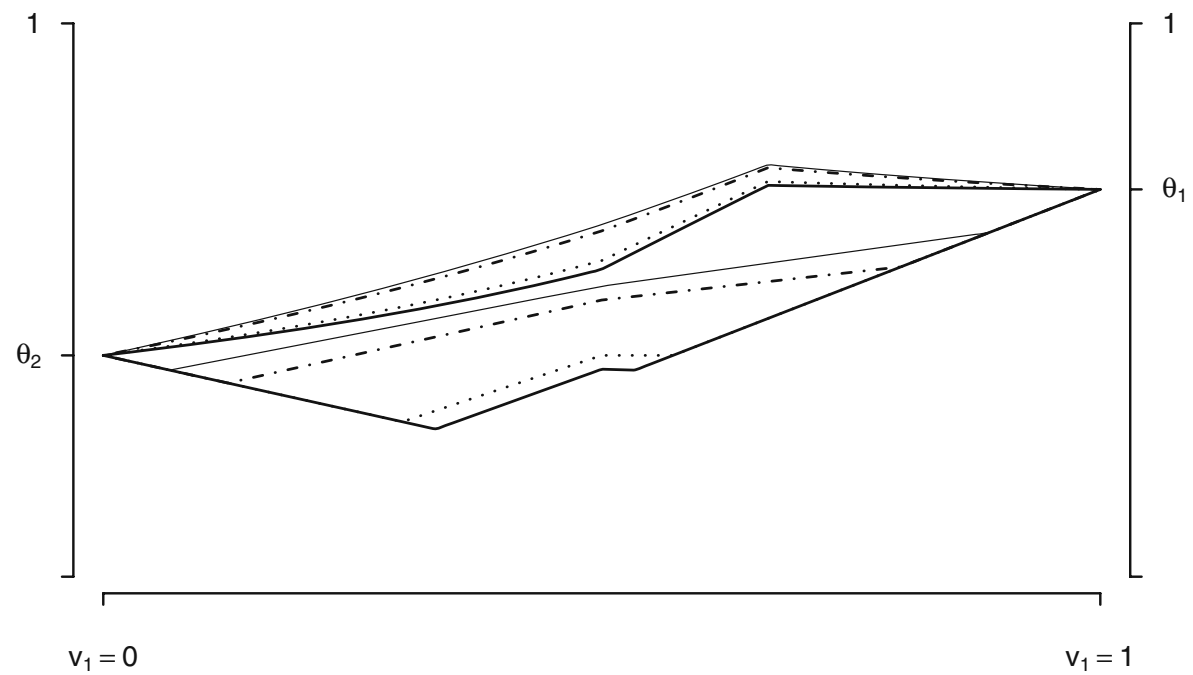

Fig. 4 Upper and lower bounds for $\theta(\boldsymbol{v})$ as in Theorem 9 given $\phi=0.75$ (thick line), $\phi=0.8$ (dotted line), $\phi=1$ (dash-dotted line) and $\phi=1.05$ (thin line) for $\theta_{1}=0.7, \theta_{2}=0.4$.

As a consequence of Theorem 5 the case $\tilde{\phi}=1$ is restricted to identical marginal dependence of all $D$ series such that $\theta_{1}=\ldots=\theta_{D}$, cf. Example 1 . It also follows from Theorem 5 that Proposition 2.1 (2) in Martins and Ferreira (2005b) where $\tilde{G}$ is assumed to have totally dependent margins (i.e. $\tilde{\phi}=1$ ) loses generality and must be restricted to the special situation where $\theta_{1}=\ldots=$ $\theta_{D}$. The case is addressed by the following corollary with $A$ defined as above.

Corollary 2 If $\tilde{\phi}_{A}=1$ then $\theta_{m}=\theta_{n}$ for all $m, n \in A$, and $\theta(\boldsymbol{v})=\theta_{m}, \boldsymbol{v} \in \mathbb{S}_{|A|}$.

Let now $\tilde{\phi}$ be given. Theorem 6 below shows that the full set of possible dependence functions $l$ compatible with $\theta_{1}, \ldots, \theta_{D}$ is not necessarily admissible for all possible values of the extremal coefficient $\tilde{\phi}$ and vice versa. Equivalently, Theorem 6 extends the set of properties of $\phi=l(\boldsymbol{\theta 1})$ given in Martins and Ferreira (2005a) by an improved upper bound related to $\tilde{\phi}$.

Theorem 6 Let $A$ be a subset of $\{1, \ldots, D\}$ with at least two elements. Then, for any stationary max-stable process with univariate extremal indices $\theta_{d} \in$ $(0,1]$ for all $d \in A$ and extremal index $\tilde{\phi}_{A}$ the adjusted extremal coefficient $\phi_{A}$ is limited by the sharp bounds

$$
\max _{d \in A} \theta_{d} \leq l\left(\boldsymbol{\theta} \boldsymbol{e}_{A}\right)=\phi_{A} \leq \min \left\{\sum_{d \in A} \theta_{d}, \tilde{\phi}_{A}-\max _{d \in A}\left(1-\theta_{d}\right)\right\} .
$$


Proof Let $\phi=\phi_{A}$ and $\tilde{\phi}=\tilde{\phi}_{A}$ and let us restrict to an $|A|$-variate $M_{4}$ process where $|A|>1$ by assumption. It is a well-known property of any dependence function that $\max _{d} \theta_{d} \leq l(\boldsymbol{\theta 1}) \leq \sum_{d} \theta_{d}$, see (L1). Further, $l(\boldsymbol{\theta 1}) \leq \tilde{l}(\mathbf{1})-$ $\max _{d}\left(1-\theta_{d}\right)$ is a direct consequence of Theorem 3 (1) and (2), and hence a closer bound for $\phi$ is given if $\tilde{\phi}-\max _{d}\left(1-\theta_{d}\right)<\sum_{d} \theta_{d}$.

1. We first give example processes reaching the bounds for the case $\tilde{\phi}<$ $\sum_{d} \theta_{d}+\max _{d}\left(1-\theta_{d}\right)$. Consider the $M_{4}$ process $A$ where $I=\{1,2\}, a_{1 d d}=$ $c \theta_{d}, a_{21 d}=(1-c) \theta_{d}, a_{2 k d}=\left(1-\theta_{d}\right) / K$ for $k=2, \ldots, K+1$ and $c \in[0,1)$. Here, $a_{21 d}>0$, and $K$ is chosen such that $a_{21 d} \geq a_{2 k d}$ for all $k$ and $d$. Further, let $B$ be the $M_{4}$ process where $I=\{1, \ldots, D+1\}, b_{d 1 d}=c \theta_{d}, b_{D+1,1, d}=$ $a_{21 d}, b_{D+1, k, d}=a_{2 k d}, k=2, \ldots, K+1, c \in[0,1)$. Now, for

$$
c=\frac{\tilde{\phi}-\max _{d} \theta_{d}-\max _{d}\left(1-\theta_{d}\right)}{\sum_{d} \theta_{d}-\max _{d} \theta_{d}} \in[0,1)
$$

$\tilde{\phi}$ is attained for both processes. Also, $A$ reaches the lower bound and $B$ reaches the upper bound for $\phi$.

2. We now consider the case $\tilde{\phi} \geq \sum_{d} \theta_{d}+\max _{d}\left(1-\theta_{d}\right)$. Let $A$ be the $R S$ process where $I=\{1\}, F=\{1,2\}, a_{1 d d}=\theta_{d}, \alpha_{1 d d}=c\left(1-\theta_{d}\right), \alpha_{21 d}=$ $(1-c)\left(1-\theta_{d}\right), c \in[0,1]$. Further, consider the $R S$ process $B$ where $I=$ $\{1, \ldots, D\}, F=\{1,2\}, b_{d 1 d}=\theta_{d}, \beta_{1 d d}=\alpha_{1 d d}, \beta_{21 d}=\alpha_{21 d}$. Now, for

$$
c=\frac{\tilde{\phi}-\sum_{d} \theta_{d}-\max _{d}\left(1-\theta_{d}\right)}{\sum_{d}\left(1-\theta_{d}\right)-\max _{d}\left(1-\theta_{d}\right)} \in[0,1]
$$

$\tilde{\phi}$ is attained for both processes where $A$ reaches the lower bound and $B$ reaches the upper bound for $\phi$.

With respect to the behavior of the multivariate extremal index we have the following corollary.

Corollary 3 For any stationary max-stable process with univariate extremal indices $\theta_{d} \in(0,1], d=1, \ldots, D$, and extremal coefficient $\tilde{\phi}$ the multivariate extremal index is bounded at $\mathbf{1}$ by

$$
\frac{\max _{d} \theta_{d}}{\tilde{\phi}} \leq \theta(\mathbf{1})=\theta\left(D^{-1} \mathbf{1}\right) \leq \frac{\min \left\{\tilde{\phi}-\max _{d}\left(1-\theta_{d}\right), \sum_{d} \theta_{d}\right\}}{\tilde{\phi}} .
$$

In the following three theorems we generalize the above corollary for $D=2$, i.e. new bounds for the entire multivariate extremal index function are given for fixed $\tilde{\phi}$ and $\phi$, respectively. Due to the complex interdependencies of higher order dependence functions (Schlather and Tawn 2002) corresponding bounds for $D \geq 3$ are not known yet. From the following theorems note that, in particular, for $\tilde{\phi}=\sum_{d} \theta_{d}+\max _{d}\left(1-\theta_{d}\right)$ in Theorem 7 the upper bound may 
not be improved as compared to Theorem 4 whereas for $\tilde{\phi}=D$ in Theorem 8 the lower bound is unchanged. We state the following example for reference in Theorems 7 and 9.

Example 2 Let $\mathcal{X}=\mathcal{X}\left(\theta_{1}, \theta_{2}, \phi_{\mathcal{X}}\right)$ be the class of $R S$ processes $X$ with coefficients $x_{j k d}, j \in I, k \in \mathbb{Z}, d=1,2$, and $\chi_{j k d}, j \in F, 0 \leq k \leq N_{j}, d=1,2$, such that $l\left(\boldsymbol{\theta} \boldsymbol{e}_{d} \mid X\right)=\theta_{d}$ and $l(\boldsymbol{\theta 1} \mid X)=\phi_{X} \leq \phi_{\mathcal{X}}$. Consider $X^{*}\left(\theta_{1}, \theta_{2}, \phi_{\mathcal{X}}\right) \in \mathcal{X}$ with $I=\{1,2,3\}, F=\{1\}, x_{d 1 d}^{*}=\phi_{\mathcal{X}}-\theta_{3-d}, x_{31 d}^{*}=\sum_{d} \theta_{d}-\phi_{\mathcal{X}}$ and $\chi_{11 d}^{*}=$ $1-\theta_{d}$, where $x_{j k d} \geq 0$. Here, $\phi_{X^{*}}=\phi_{\mathcal{X}}$. Now, using the results of Theorem 3 ,

$$
\begin{aligned}
l\left(\boldsymbol{\theta} v \mid X^{*}\right) & =\tilde{l}_{\theta}\left(\boldsymbol{v} \mid X^{*}\right)=\sum_{d}\left(\phi_{X^{*}}-\theta_{3-d}\right) v_{d}+\left(\sum_{d} \theta_{d}-\phi_{X^{*}}\right) \max _{d} v_{d}, \\
\tilde{l}_{1-\theta}\left(\boldsymbol{v} \mid X^{*}\right) & =\tilde{l}_{1-\theta, \min }(\boldsymbol{v}), \\
\tilde{l}\left(\boldsymbol{v} \mid X^{*}\right) & =\tilde{l}_{\theta}\left(\boldsymbol{v} \mid X^{*}\right)+\tilde{l}_{1-\theta}\left(\boldsymbol{v} \mid X^{*}\right) .
\end{aligned}
$$

Then, $l\left(\boldsymbol{\theta 1} \mid X^{*}\right) \geq l(\boldsymbol{\theta 1} \mid X)$, and in conjunction with convexity and piecewise linearity we have

$$
l\left(\theta v \mid X^{*}\right) \geq l(\theta v \mid X) \quad \text { for all } X \in \mathcal{X} .
$$

Now, by Theorem 3 (2)

$$
\frac{l(\boldsymbol{\theta} \boldsymbol{v} \mid X)}{\tilde{l}(\boldsymbol{v} \mid X)} \leq \frac{l(\boldsymbol{\theta} \boldsymbol{v} \mid X)}{l(\boldsymbol{\theta} \boldsymbol{v} \mid X)+\tilde{l}_{1-\theta, \min }(\boldsymbol{v})} \leq \frac{l\left(\boldsymbol{\theta} \boldsymbol{v} \mid X^{*}\right)}{l\left(\boldsymbol{\theta} \boldsymbol{v} \mid X^{*}\right)+\tilde{l}_{1-\theta, \min }(\boldsymbol{v})}=\theta\left(\boldsymbol{v} \mid X^{*}\right)
$$

for all $X \in \mathcal{X}$ using the same argumentation as in the proof of Theorem 4 for the second inequality.

Theorem 7 Let $D=2$ and $\Theta\left(\theta_{1}, \theta_{2}, \tilde{\phi}\right)$ be the closed set of multivariate extremal index functions $\theta$ of all stationary max-stable processes with univariate extremal indices $\theta_{1}, \theta_{2} \in(0,1]$ and extremal coefficient $\tilde{\phi}$. Define

$$
\theta_{\text {sup }}: \mathbb{S}_{2} \rightarrow(0,1], \boldsymbol{v} \mapsto \sup _{\theta \in \Theta\left(\theta_{1}, \theta_{2}, \tilde{\phi}\right)} \theta(\boldsymbol{v}) .
$$

1. If $\tilde{\phi} \leq \sum_{d} \theta_{d}+\max _{d}\left(1-\theta_{d}\right)$, then $\theta_{\text {sup }} \in \Theta$ and

$$
\theta_{\text {sup }}(\boldsymbol{v})=\left(1+\frac{\max _{d}\left(1-\theta_{d}\right) v_{d}}{\sum_{d}\left(\phi^{*}-\theta_{3-d}\right) v_{d}+\left(\theta_{1}+\theta_{2}-\phi^{*}\right) \max _{d} v_{d}}\right)^{-1},
$$

where $\phi^{*}=\tilde{\phi}-\max _{d}\left(1-\theta_{d}\right)$.

2. If $\tilde{\phi}>\sum_{d} \theta_{d}+\max _{d}\left(1-\theta_{d}\right)$, then $\theta_{\text {sup }} \in \Theta$ iff $\tilde{\phi}=D$, and

$$
\theta_{\text {sup }}(\boldsymbol{v})=\left(1+\frac{\min _{d}\left\{\max \left\{\left(1-\theta_{d}\right) v_{d},(2-\tilde{\phi}) v_{3-d}\right\}+\left(\tilde{\phi}-1-\theta_{3-d}\right) v_{3-d}\right\}}{\sum_{d} \theta_{d} v_{d}}\right)^{-1} .
$$


Proof The proof of Theorem 2 shows that $\Theta\left(\theta_{1}, \theta_{2}, \tilde{\phi}\right)$ is closed. Let $\mathcal{B}=$ $\mathcal{B}\left(\theta_{1}, \theta_{2}, \tilde{\phi}\right)$ be the class of $R S$ processes $B$ where $l\left(\boldsymbol{\theta} \boldsymbol{e}_{d} \mid B\right)=\theta_{d}$ and $\tilde{l}(\mathbf{1} \mid B)=\tilde{\phi}$.

1. For $\mathcal{X}$ as in Example 2, $\mathcal{B}\left(\theta_{1}, \theta_{2}, \tilde{\phi}\right) \subset \mathcal{X}\left(\theta_{1}, \theta_{2}, \tilde{\phi}-\max _{d}\left(1-\theta_{d}\right)\right)$ by Theorem 6. Now, it is easily verified that $X^{*}\left(\theta_{1}, \theta_{2}, \tilde{\phi}-\max _{d}\left(1-\theta_{d}\right)\right) \in \mathcal{B}$.

2. Using the same notation as in Theorems 3 and 4 we have that $\mathcal{B}\left(\theta_{1}, \theta_{2}, \tilde{\phi}\right) \subset$ $\mathcal{A}\left(\theta_{1}, \theta_{2}\right)$, and hence

$$
\theta(\boldsymbol{v} \mid B) \leq \frac{l_{\max }(\boldsymbol{\theta v})}{l_{\max }(\boldsymbol{\theta} \boldsymbol{v})+\tilde{l}_{1-\theta, \min }(\boldsymbol{v})}=: \theta_{U, 1}(\boldsymbol{v}) .
$$

Further, from $\tilde{l}(\mathbf{1} \mid B)=\tilde{\phi}$ it follows by convexity and piecewise linearity that $\tilde{l}(\boldsymbol{v} \mid B) \geq \sum_{d} v_{d}-\max _{d}\left\{\left(2 \sum_{d} v_{d}-\tilde{\phi}\right) v_{d}\right\}=1-(2-\tilde{\phi}) \max _{d} v_{d}$, and hence, a second upper bound is given by

$$
\theta_{U, 2}(\boldsymbol{v})=\frac{l_{\max }(\boldsymbol{\theta v})}{1-(2-\tilde{\phi}) \max _{d} v_{d}} .
$$

Now, we show that

$$
\begin{aligned}
\min & \left\{\theta_{U, 1}(\boldsymbol{v}), \theta_{U, 2}(\boldsymbol{v})\right\} \\
& =\frac{l_{\max }(\boldsymbol{\theta v})}{\max \left\{l_{\max }(\boldsymbol{\theta} \boldsymbol{v})+\tilde{l}_{1-\theta, \min }(\boldsymbol{v}), 1-(2-\tilde{\phi}) \max _{d} v_{d}\right\}} \\
& =\frac{l_{\max }(\boldsymbol{\theta} \boldsymbol{v})}{\min _{d}\left\{\max \left\{\left(1-\theta_{d}\right) v_{d},(2-\tilde{\phi}) v_{3-d}\right\}+\left(\tilde{\phi}-1-\theta_{3-d}\right) v_{3-d}\right\}+\sum_{d} \theta_{d} v_{d}} \\
& =\frac{l_{\max }(\boldsymbol{\theta} \boldsymbol{v})}{\min \left\{\tilde{l}_{1}(\boldsymbol{v}), \tilde{l}_{2}(\boldsymbol{v})\right\}}=\theta_{\text {sup }}(\boldsymbol{v})
\end{aligned}
$$

is a sharp upper bound for valid dependence functions $\tilde{l}_{1}$ and $\tilde{l}_{2}$ consistent with $l(\boldsymbol{\theta} v)=l_{\max }(\boldsymbol{\theta} v)$. Here, the second equation follows after some lengthy but elementary calculations. Note that $\theta_{\text {sup }}$ is reached piecewise by the $R S$ example processes $B_{m} \in \mathcal{B}, m=1$, 2, where $b_{m, d 1 d}=\theta_{d}, \beta_{m, 11 m}=$ $1-\theta_{m}, \beta_{m,(1,1,3-m)}=2-\tilde{\phi}, \beta_{m,(1,2,3-m)}=\tilde{\phi}-\tilde{\sigma}_{\tilde{\phi}}-\theta_{3-m}$, and $\beta_{m,(1,2,3-m)} \geq$ $0, m=1,2$, by assumption on $\tilde{\phi}$. Finally, for $\tilde{\phi}<D$ by lack of convexity of $\min \left\{\tilde{l}_{1}, \tilde{l}_{2}\right\}$ we have that $\theta_{\text {sup }} \notin \Theta$ whereas for $\tilde{\phi}=D$ it holds that $\tilde{l}_{1}=\tilde{l}_{2}$, and $\theta_{\text {sup }} \in \Theta$.

Except for specific parameter values the lower bound for $\theta(\boldsymbol{v})$ given $\theta_{1}, \theta_{2}$ and $\tilde{\phi}$ to be discussed next and represented in Fig. 3 appears to be of a more complex form than the upper bound in the last theorem. For a motivation of the structure of the processes involved we first give the following example process. Namely, for the complex case when $\tilde{\phi}<\sum_{d} \theta_{d}+\max _{d}\left(1-\theta_{d}\right)$, see the theorem below, it will turn out to be a simple member of two classes of 
processes reaching the lower bound pointwise for certain values of $\boldsymbol{v} \in \mathbb{S}_{2}$. Further, for the remaining values of $v \in \mathbb{S}_{2}$ the process reaches the lower bound piecewise. In the following example and in Theorem 8 we will make use of a certain partition of $\boldsymbol{v} \in \mathbb{S}_{2}$. To this end let for $\theta_{1} \geq \theta_{2}$

$$
\begin{gathered}
V_{1}=\left[0, \frac{\tilde{\phi}-1+\theta_{2}-\theta_{1}}{2 \tilde{\phi}-2+\theta_{2}-\theta_{1}}\right], \quad V_{3}=\left[\frac{1}{2}, 1\right], \quad V_{2}=[0,1] \backslash\left(V_{1} \cup V_{3}\right), \\
V_{2,2}=\left[\frac{\theta_{2}}{\theta_{1}+\theta_{2}}, \frac{1}{2}\right], \quad V_{2,1}=V_{2} \backslash V_{2,2} .
\end{gathered}
$$

Example 3 For $D=2, \theta_{1}>\theta_{2}, \tilde{\phi}<1+\theta_{1}$ and $\boldsymbol{v}^{*} \in \mathbb{S}_{2}$ let $C=C\left(\theta_{1}, \theta_{2}, \tilde{\phi}, v_{1}^{*}\right)$ be the $M_{4}$ process with coefficients $c_{j k d}, j \in I=\{1,2\}, k \in \mathbb{N}, d=1,2$, where

$$
\begin{aligned}
& c_{11 d}=\left(1-q_{d}\right) \theta_{d}, \\
& c_{2 d d}=q_{d} \theta_{d}, \\
& c_{1 k d}=\left(1-\theta_{1}\right) / K \text { for } k=2, \ldots, K+1, \\
& c_{1 k 2}=\left(\theta_{1}-\theta_{2}\right) / K \text { for } k=2+K, \ldots, 1+2 K
\end{aligned}
$$

for some $q_{d} \in[0,1)$ specified below and $K$ the smallest positive integer such that $c_{11 d} \geq c_{1 k d}$ for $k>2, d=1,2$. Further,

$$
\begin{aligned}
& q_{2}=\frac{\tilde{\phi}-1+\theta_{2}-\theta_{1}}{\theta_{2}} \in[0,1), \\
& q_{1}\left(\boldsymbol{v}^{*}\right)= \begin{cases}\frac{\tilde{\phi}-1}{\theta_{1}} \in(0,1), & v_{1}^{*} \in V_{1}, \\
\frac{\theta_{2}}{\theta_{1}} q_{2}\left(1 / v_{1}^{*}-1\right) \in[0,1), & v_{1}^{*} \in V_{2,1}, \\
q_{2}, & v_{1}^{*} \in V_{2,2} \cup V_{3}\end{cases}
\end{aligned}
$$

such that $c_{11 d}>0$. Now, for $Z_{1}^{C}=\left\{(j, k) \in I \times \mathbb{Z}: c_{j k d}>0, d=1,2\right\}=$ $\{(1, k): k=1, \ldots, K+1\}$ and $Z_{2}^{C}=\left\{(j, k) \in Z_{1}^{C}: c_{j k 1}=\max _{k} c_{j k 1}\right\}=\{(1,1)\}$ the meaning of which will become clear in the proof of the next theorem it obviously holds that

1. $\tilde{l}(\mathbf{1} \mid C)=\tilde{\phi}$

2. $c_{j k 1}=c_{j k 2}, \quad(j, k) \in Z_{1}^{C} \backslash Z_{2}^{C}$,

3. $\sum_{(j, k) \in Z_{1}^{C} \backslash Z_{2}^{C}} c_{j k 1}=1-\theta_{1}$,

4. $c_{112}=c_{111} \frac{v_{1}^{*}}{v_{2}^{*}}+\theta_{2}-\theta_{1} \frac{v_{1}^{*}}{v_{2}^{*}} \geq c_{111} \frac{v_{1}^{*}}{v_{2}^{*}}, c_{222}=c_{211} \frac{v_{1}^{*}}{v_{2}^{*}}, v_{1}^{*} \in V_{2,1}, v_{2}^{*}=1-v_{1}^{*}$,

5. $c_{112}=c_{111} \theta_{2} / \theta_{1}, c_{222}=c_{211} \theta_{2} / \theta_{1}, \quad v_{1}^{*} \in V_{2,2} \cup V_{3}$. 
Theorem 8 Let $D=2$ and $\Theta\left(\theta_{1}, \theta_{2}, \tilde{\phi}\right)$ be the closed set of multivariate extremal index functions $\theta$ for all stationary max-stable processes with univariate extremal indices $\theta_{1}, \theta_{2} \in(0,1]$ and extremal coefficient $\tilde{\phi}$. Define

$$
\theta_{\text {inf }}: \mathbb{S}_{2} \rightarrow(0,1], \boldsymbol{v} \mapsto \inf _{\theta \in \Theta\left(\theta_{1}, \theta_{2}, \tilde{\phi}\right)} \theta(\boldsymbol{v}) .
$$

1. If $\tilde{\phi} \geq \sum_{d} \theta_{d}+\max _{d}\left(1-\theta_{d}\right)$ or $\theta_{1}=\theta_{2}$, then $\theta_{\mathrm{inf}} \in \Theta$ and

$$
\theta_{\text {inf }}(\boldsymbol{v})=\frac{\max _{d} \theta_{d} v_{d}}{\tilde{\phi}-1+(2-\tilde{\phi}) \max _{d} v_{d}}, \boldsymbol{v} \in \mathbb{S}_{2} .
$$

2. If $\theta_{1}>\theta_{2}$ and $\tilde{\phi}<\sum_{d} \theta_{d}+\max _{d}\left(1-\theta_{d}\right)=1+\theta_{1}$, then

$$
\theta_{\text {inf }}(\boldsymbol{v})= \begin{cases}\frac{\theta_{2} v_{2}}{1-(2-\tilde{\phi}) v_{1}}, & v_{1} \in V_{1}, \\ \frac{\max _{d} \theta_{d} v_{d}}{\max _{d} \theta_{d} v_{d}+\left(\tilde{\phi}-\theta_{1}\right) v_{2}}, & v_{1} \in V_{2}, \\ \frac{\theta_{1} v_{1}}{1-(2-\tilde{\phi}) v_{2}}, & v_{1} \in V_{3},\end{cases}
$$

where $v_{2}=1-v_{1}$. In particular, $\theta_{\mathrm{inf}} \notin \Theta$. The assertion for $\theta_{1}<\theta_{2}$ is given by symmetry.

Proof Again, by Proposition 1 it suffices to restrict to the respective bounds of $M_{4}$ processes.

1. For $\tilde{\phi} \geq \sum_{d} \theta_{d}+\max _{d}\left(1-\theta_{d}\right)$ and $\theta_{1}, \theta_{2} \in(0,1]$ consider a sequence of $M_{4}$ processes $A$ with $I=\{1\}$ given by $a_{1 d d}=\theta_{d}, a_{1 k d}=\frac{2-\tilde{\phi}}{K}$ for $k=D+$ $1, \ldots, D+K, a_{1 k d}=\frac{\tilde{\phi}-1-\theta_{d}}{K} \geq 0$ for $k=D+d K+1, \ldots, D+(d+1) K$ and $K$ such that $\theta_{d} \geq a_{1 k d}$ for all $k, d$. Now, $l(\boldsymbol{\theta} v \mid A)=l_{\min }(\boldsymbol{\theta v})$ and $\tilde{l}(\boldsymbol{v} \mid A)=$ $\tilde{l}_{\max }(\boldsymbol{v} \mid \tilde{\phi})$ by convexity and piecewise linearity where $\tilde{l}_{\max }(\boldsymbol{v} \mid \tilde{\phi})$ is the overall maximum of $\tilde{l}(\boldsymbol{v})$ given $\tilde{\phi}$.

For $\theta_{1}=\theta_{2}$ and $\tilde{\phi}<\sum_{d} \theta_{d}+\max _{d}\left(1-\theta_{d}\right)=1+\theta_{1}$ consider the process $C\left(\theta_{1}, \theta_{1}, \tilde{\phi}, 0\right)$ in Example 3. We have that $l(\boldsymbol{\theta} v \mid C)=l_{\min }(\boldsymbol{\theta} \boldsymbol{v})$ and $\tilde{l}(\boldsymbol{v} \mid C)=$ $\tilde{l}_{\max }(\boldsymbol{v} \mid \tilde{\phi})$ by convexity and piecewise linearity.

2. We consider separately the four subsets $V_{1}, V_{2,1}, V_{2,2}$ and $V_{3}$ for $v_{1}$ with $v \in \mathbb{S}_{2}$.

(1) For $v_{1} \in V_{1}$ consider the process $C\left(\theta_{1}, \theta_{2}, \tilde{\phi}, v_{1}\right)$ in Example 3. Now, $l(\boldsymbol{\theta} \boldsymbol{v} \mid$ $C)=\theta_{2} v_{2}=l_{\min }(\boldsymbol{\theta} \boldsymbol{v})$ and $\tilde{l}(\boldsymbol{v} \mid C)=1-(2-\tilde{\phi}) v_{1}=\tilde{l}_{\max }(\boldsymbol{v} \mid \tilde{\phi}), v_{1} \in V_{1}$.

(2) Throughout this part we fix $\boldsymbol{v} \in \mathbb{S}_{2}$ with $v_{1} \in V_{2,1}$. Let $\mathcal{B}=\mathcal{B}\left(\theta_{1}, \theta_{2}, \tilde{\phi}\right)$ be the class of $M_{4}$ processes $B$ with coefficients $b_{j k d}, j \in I, k \in \mathbb{Z}, d=1,2$, where $l\left(\boldsymbol{\theta} \boldsymbol{e}_{d} \mid B\right)=\theta_{d}$ and $\tilde{l}(\mathbf{1} \mid B)=\tilde{\phi}$. We will show that a process $C$ with $l(\boldsymbol{\theta} v \mid C)=l_{\min }(\boldsymbol{\theta} v)$ exists such that for all $B \in \mathcal{B}$ the inequality $\tilde{l}(\boldsymbol{v} \mid B)-$ $\tilde{l}(\boldsymbol{v} \mid C) \leq l(\boldsymbol{\theta} v \mid B)-l(\boldsymbol{\theta} \boldsymbol{v} \mid C)$ holds. Then, $l(\boldsymbol{\theta} \boldsymbol{v} \mid C) / \tilde{l}(\boldsymbol{v} \mid C)=\theta_{\text {inf }}(\boldsymbol{v})$ by 
discussion of the mapping $x \mapsto \frac{x+a}{x+b}, 0 \leq a \leq b, x \geq 0$. For the calculation of $\tilde{l}$ it will be advantageous to replace the double index $(j, k) \in I \times \mathbb{Z}$ by a single one, $m \in \mathbb{Z}$. More precisely, let $f: I \times \mathbb{Z} \rightarrow \mathbb{Z},(j, k) \mapsto f(j, k)$ be an arbitrary bijective mapping and define $b_{m d}=b_{f^{-1}(m), d}$. Then,

$$
\tilde{l}(\boldsymbol{v} \mid B)=\sum_{m \in \mathbb{Z}} \max _{d} b_{m d} v_{d}=\sum_{d} v_{d}-m(\boldsymbol{v} \mid B),
$$

with $m(\boldsymbol{v} \mid B)=\sum_{m \in \mathbb{Z}} \min _{d} b_{m d} v_{d} \geq 0$.

Let $\pi$ be the projection $\pi: I \times \mathbb{Z} \rightarrow I,(j, k) \mapsto j$, and define $g:=$ $\pi \circ f^{-1}: \mathbb{Z} \rightarrow I, m \mapsto g(m)=j$. Let $b_{j d}^{*}=b_{j, k_{j d}^{*}, d}$, for $k_{j d}^{*} \in \arg \max _{k} b_{j k d}$, $j \in I, d=1,2$. Since the set of $M_{4}$ processes $B$ for which $k_{j d}^{*}$ is unique is a dense subset of $\mathcal{B}$ we may assume uniqueness of $k_{j d}^{*}$. Let $Z_{1}^{B}=\{m \in \mathbb{Z}$ : $\left.b_{m d}>0, d=1,2\right\}$ such that

$$
\sum_{m \in \mathbb{Z}} \min _{d} b_{m d}=\sum_{m \in Z_{1}^{B}} \min _{d} b_{m d}=2-\tilde{\phi}=m(\mathbf{1} \mid B) .
$$

Let $Z_{2}^{B}=\left\{m \in Z_{1}^{B}: b_{m 1}=b_{g(m), 1}^{*}\right\}$. Now,

$$
\sum_{m \in Z_{1}^{B} \backslash Z_{2}^{B}} b_{m 1} \leq 1-\theta_{1}
$$

such that

$$
\sum_{m \in Z_{1}^{B} \backslash Z_{2}^{B}} \min _{d} b_{m d}=1-\theta_{1}-\mu
$$

for $0 \leq \mu \leq \min \left\{1-\theta_{1}, \tilde{\phi}-1+\theta_{2}-\theta_{1}\right\}$, where the latter follows from the fact that

$$
1-\theta_{1}-\mu=2-\tilde{\phi}-\sum_{m \in Z_{2}^{B}} \min _{d} b_{m d}
$$

by Eqs. 10 and 8 , and $\sum_{m \in Z_{2}^{B}} \min _{d} b_{m d} \leq \min _{d} \sum_{m \in Z_{2}^{B}} b_{m d} \leq \min _{d} \theta_{d}=\theta_{2}$. Note that from $\tilde{\phi}<1+\theta_{1}$, Eq. 8 and Ineq. 9 it follows that $Z_{2}^{B} \neq \emptyset$. Now, with

$$
\sum_{m \in Z_{1}^{B} \backslash Z_{2}^{B}} \min _{d} b_{m d} v_{d} \geq \sum_{m \in Z_{1}^{B} \backslash Z_{2}^{B}} \min _{d} b_{m d} \min _{d} v_{d}=\left(1-\theta_{1}-\mu\right) v_{1}
$$

we get

$$
m(\boldsymbol{v} \mid B) \geq \sum_{m \in Z_{2}^{B}} \min _{d} b_{m d} v_{d}+\left(1-\theta_{1}-\mu\right) v_{1} .
$$


We consider the (disjoint) decomposition $Z_{2}^{B}=Z_{2,1}^{B} \cup Z_{2,2}^{B} \cup Z_{2,3}^{B}$ where $m \in Z_{2,1}^{B}$ if $b_{m 1}<b_{m 2}, m \in Z_{2,2}^{B}$ if $b_{m 2} \leq b_{m 1} \leq b_{m 2} v_{2} / v_{1}$ and $m \in Z_{2,3}^{B}$ else. Now,

$$
\begin{aligned}
& \min _{d} b_{m d}=\left\{\begin{array}{l}
b_{m 1}=b_{m 2}-\xi_{m}, m \in Z_{2,1}^{B} \\
b_{m 2}, m \in Z_{2,2}^{B} \cup Z_{2,3}^{B},
\end{array}\right. \\
& \min _{d}\left\{b_{m d} v_{d}\right\}=\left\{\begin{array}{l}
b_{m 1} v_{1}, m \in Z_{2,1}^{B} \cup Z_{2,2}^{B} \\
b_{m 2} v_{2}=\left(b_{m 1}-\eta_{m}\right) v_{1}, m \in Z_{2,3}^{B},
\end{array}\right. \\
& \max _{d}\left\{b_{g(m), d}^{*} v_{d}\right\}=\left\{\begin{array}{l}
\left(b_{m 2}+\kappa_{m}\right) v_{2}, m \in Z_{2,1}^{B} \cup Z_{2,2}^{B} \\
\left(b_{m 2}+\kappa_{m}\right) v_{2}+\max \left\{0, \eta_{m} v_{1}-\kappa_{m} v_{2}\right\}, m \in Z_{2,3}^{B},
\end{array}\right.
\end{aligned}
$$

where $\xi_{m}=b_{m 2}-b_{m 1}>0, m \in Z_{2,1}^{B}, \kappa_{m}=b_{g(m), 2}^{*}-b_{m 2} \geq 0, m \in Z_{2}^{B}$, and $0<\eta_{m}=b_{g(m), 1}^{*}-b_{m 2} v_{2} / v_{1}=b_{m 1}-b_{m 2} v_{2} / v_{1} \leq 1-b_{m 2} v_{2} / v_{1}, m \in$ $Z_{2,3}^{B}$. Let

$$
\begin{aligned}
s_{m 1} & =\sum_{j \in I \backslash\left\{g(m): m \in Z_{2}^{B}\right\}} b_{j 1}^{*}=\theta_{1}-\sum_{m \in Z_{2}^{B}} b_{m 1} \\
& =\theta_{1}-\left\{\sum_{m \in Z_{2}^{B}} \min _{d} b_{m d} v_{d} / v_{1}+\sum_{m \in Z_{2,3}^{B}} \eta_{m}\right\}, \\
s_{m 2} & =\sum_{j \in I \backslash\left\{g(m): m \in Z_{2}^{B}\right\}} b_{j 2}^{*}=\theta_{2}-\sum_{m \in Z_{2}^{B}} b_{g(m), 2}^{*} \\
& =\theta_{2}-\left\{\sum_{m \in Z_{2}^{B}} \min _{d} b_{m d}+\sum_{m \in Z_{2,1}^{B}} \xi_{m}+\sum_{m \in Z_{2}^{B}} \kappa_{m}\right\} .
\end{aligned}
$$

Then,

$$
\begin{aligned}
l(\boldsymbol{\theta} \boldsymbol{v} \mid B)= & \sum_{m \in Z_{2}^{B}} \max _{d}\left\{b_{g(m), d}^{*} v_{d}\right\}+\sum_{j \in I \backslash\left\{g(m): m \in Z_{2}^{B}\right\}} \max _{d}\left\{b_{j d}^{*} v_{d}\right\} \\
\geq & \sum_{m \in Z_{2}^{B}} b_{m 2} v_{2}+\sum_{m \in Z_{2}^{B}} \kappa_{m} v_{2} \\
& +\sum_{m \in Z_{2,3}^{B}} \max \left\{0, \eta_{m} v_{1}-\kappa_{m} v_{2}\right\}+s_{m 1} v_{1} .
\end{aligned}
$$


Let $\mathcal{C} \subset \mathcal{B}$ be the class of $M_{4}$ processes $C$ with coefficients $c_{j k d}$ where

$$
\begin{gathered}
c_{j 2}^{*}=\frac{v_{1}}{v_{2}} c_{j 1}^{*}+\gamma_{j}, \quad j \in I, \gamma_{j} \geq 0, \\
c_{m 1}=c_{m 2}, \quad m \in Z_{1}^{C} \backslash Z_{2}^{C}, \\
\sum_{m \in Z_{1}^{C} \backslash Z_{2}^{C}} c_{m 1}=1-\theta_{1}, \\
c_{m 2}=c_{g(m), 2}^{*}=\frac{v_{1}}{v_{2}} c_{m 1}+\gamma_{g(m)} \leq c_{m 1}, m \in Z_{2}^{C}, \quad \text { and } \\
\sum_{m \in Z_{2}^{C}} \gamma_{g(m)}=\sum_{j \in I} \gamma_{j}=\theta_{2}-\frac{v_{1}}{v_{2}} \theta_{1} .
\end{gathered}
$$

Here, Eq. 18 replaces the corresponding Ineq. 9 above. In particular, $\mathcal{C}$ is not empty by Example 3. Since $\sum_{m \in Z_{1}^{C} \backslash Z_{2}^{C}} \min _{d} c_{m d}=1-\theta_{1}$ by Eqs. 17 and 18 it holds with Eqs. 19 and 8 that

$$
\sum_{m \in Z_{2}^{C}} \min _{d} c_{m d}=\sum_{m \in Z_{2}^{C}} c_{m 2}=1-\tilde{\phi}+\theta_{1} .
$$

By the left hand side of Eq. 19 we now get with Eq. 21 that

$$
\begin{aligned}
\sum_{m \in Z_{2}^{C}} c_{m 1} v_{1} & =\left(1-\tilde{\phi}+\theta_{1}-\sum_{m \in Z_{2}^{C}} \gamma_{m}\right) v_{2} \\
& =\left(1-\tilde{\phi}-\theta_{2}\right) v_{2}+\theta_{1}, \quad \boldsymbol{v} \in \mathbb{S}_{2},
\end{aligned}
$$

where Eq. 22 follows from Eq. 20. Further, by Eqs. 19 and 17,

$$
\begin{aligned}
m(\boldsymbol{v} \mid C)=\sum_{m \in Z_{1}^{C}} \min _{d} c_{m d} v_{d} & =\sum_{m \in Z_{2}^{C}} c_{m 1} v_{1}+\sum_{m \in Z_{1}^{C} \backslash Z_{2}^{C}} c_{m 1} v_{1} \\
& =1-\left(\tilde{\phi}-\theta_{1}+\theta_{2}\right) v_{2},
\end{aligned}
$$

where Eq. 24 follows with Eqs. 22 and 18. To conclude the proof we will make use of the following four results.

First, by Eq. 16 and the definition of $s_{m 2}$ we have

$$
l(\boldsymbol{\theta} \boldsymbol{v} \mid C)=l_{\min }(\boldsymbol{\theta} \boldsymbol{v})=\theta_{2} v_{2}=s_{m 2} v_{2}+\sum_{m \in Z_{2}^{B}} b_{g(m), 2}^{*} v_{2}, \quad v_{1} \in V_{2,1} .
$$

Eqs. 21 and 11 imply

$$
s_{m 2}=\theta_{2}-\left\{\sum_{m \in Z_{2}^{C}} \min _{d} c_{m d}+\mu+\sum_{m \in Z_{2,1}^{B}} \xi_{m}+\sum_{m \in Z_{2}^{B}} \kappa_{m}\right\} .
$$


Further, applying Eq. 19 twice and Eq. 20 yields

$$
\sum_{m \in Z_{2}^{C}} \min _{d} c_{m d} v_{2}=\sum_{m \in Z_{2}^{C}} c_{m 1} v_{1}+\theta_{2} v_{2}-\theta_{1} v_{1} .
$$

Finally, by Eqs. 23 and 18 and Ineq. 13

$$
m(\boldsymbol{v} \mid C)-m(\boldsymbol{v} \mid B) \leq \sum_{m \in Z_{2}^{C}} c_{m 1} v_{1}-\sum_{m \in Z_{2}^{B}} \min _{d} b_{m d} v_{d}+\mu v_{1} .
$$

Now,

$$
\begin{aligned}
& l(\boldsymbol{\theta} \boldsymbol{v} \mid B)-l(\boldsymbol{\theta} \boldsymbol{v} \mid C)=l(\boldsymbol{\theta} \boldsymbol{v} \mid B)-\theta_{2} v_{2} \\
& \geq s_{m 1} v_{1}-s_{m 2} v_{2}+\sum_{m \in Z_{2,3}^{B}}\left(\eta_{m} v_{1}-\kappa_{m} v_{2}\right) \\
& =\sum_{m \in Z_{2}^{C}} c_{m 1} v_{1}-\sum_{m \in Z_{2}^{B}} \min _{d} b_{m d} v_{d}+\mu v_{2}+\sum_{m \in Z_{2,1}^{B}} \xi_{m} v_{2} \\
& \quad+\sum_{m \in Z_{2}^{B}} \kappa_{m} v_{2}-\sum_{m \in Z_{2,3}^{B}} \kappa_{m} v_{2} \\
& \geq m(\boldsymbol{v} \mid C)-m(\boldsymbol{v} \mid B),
\end{aligned}
$$

where Ineq. 29 holds with Ineq. 15 and Eq. 25, Eq. 30 holds with Eqs. 26, 27 and the definition of $s_{m 1}$, and Ineq. 31 finally follows from Ineq. 28.

(3) Let $\boldsymbol{v} \in \mathbb{S}_{2}$ with $v_{1} \in V_{2,2}$ be fixed. The proof is similar to that of part (2) and uses the same notation where possible. Let now $\mathcal{C}$ be the class of $M_{4}$ processes $C$ with coefficients $c_{j k d}$ where

$$
c_{j 2}^{*}=\frac{v_{1}}{v_{2}} c_{j 1}^{*}-\gamma_{j}, \quad j \in I, \gamma_{j} \geq 0, \sum_{j \in I} \gamma_{j}=\frac{v_{1}}{v_{2}} \theta_{1}-\theta_{2},
$$

and Eqs. 17 and 18 hold. From Eq. 32 we get for $m \in Z_{2}^{C}$ that

$$
c_{m 2}=\frac{v_{1}}{v_{2}} c_{m 1}-\gamma_{g(m)}-\varepsilon_{m} \leq c_{m 1},
$$

where $0 \leq \varepsilon_{m} \leq \frac{v_{1}}{v_{2}} c_{m 1}-\gamma_{g(m)}$ accounts for the fact that $c_{m 2} \leq c_{g(m), 2}^{*}$. Again, $\mathcal{C}$ is not empty by Example 3 , where $\gamma_{j}=c_{j 1}^{*}\left(v_{1} / v_{2}-\theta_{2} / \theta_{1}\right)$ and

$$
l(\boldsymbol{\theta} \boldsymbol{v} \mid \boldsymbol{C})=l_{\min }(\boldsymbol{\theta} \boldsymbol{v})=\theta_{1} v_{1}
$$

by Eq. 32. From Eq. 33 it follows that $c_{m 1} v_{1} \geq c_{m 2} v_{2}, v_{1} \in V_{2,2}$, and hence

$$
m(\boldsymbol{v} \mid C)=\sum_{m \in Z_{2}^{C}} c_{m 2} v_{2}+\sum_{m \in Z_{1}^{C} \backslash Z_{2}^{C}} c_{m 1} v_{1} .
$$

Further, following the argumentation there, Eq. 21 holds with Eq. 33 instead of Eq. 19. Now, with Eqs. 35 and 18 it follows that

$$
m(\boldsymbol{v} \mid C)=\left(1-\tilde{\phi}+\theta_{1}\right) v_{2}+\left(1-\theta_{1}\right) v_{1} .
$$


Consider again the class $\mathcal{B}$ as in part (2). Using the above decomposition of $Z_{2}^{B}$ we may write

$m(\boldsymbol{v} \mid B)=\sum_{m \in Z_{2}^{B}} b_{m 2} v_{2}-\sum_{m \in Z_{2,1}^{B} \cup Z_{2,2}^{B}}\left(b_{m 2} v_{2}-b_{m 1} v_{1}\right)+\sum_{m \in Z_{1}^{B} \backslash Z_{2}^{B}} \min _{d} b_{m d} v_{d}$.

Eq. 11 states that $1-\tilde{\phi}+\theta_{1}+\mu=\sum_{m \in Z_{2}^{B}} \min _{d} b_{m d} \leq \sum_{m \in Z_{2}^{B}} b_{m 2}$. Now, using Ineq. 12 and Ineq. 36 it follows that

$$
\begin{aligned}
\sum_{m \in Z_{2}^{B}} b_{m 2} v_{2}+\sum_{m \in Z_{1}^{B} \backslash Z_{2}^{B}} \min _{d} b_{m d} v_{d} & \geq\left(1-\tilde{\phi}+\theta_{1}+\mu\right) v_{2}+\left(1-\theta_{1}-\mu\right) v_{1} \\
& \geq\left(1-\tilde{\phi}+\theta_{1}\right) v_{2}+\left(1-\theta_{1}\right) v_{1} \\
& =m(\boldsymbol{v} \mid C),
\end{aligned}
$$

and hence

$$
m(\boldsymbol{v} \mid C)-m(\boldsymbol{v} \mid B) \leq \sum_{m \in Z_{2,1}^{B} \cup Z_{2,2}^{B}}\left(b_{m 2} v_{2}-b_{m 1} v_{1}\right) .
$$

Further, with

$$
\max _{d}\left\{b_{g(m), d}^{*} v_{d}\right\}=\left\{\begin{array}{l}
b_{m 1} v_{1}+\left(b_{m 2} v_{2}-b_{m 1} v_{1}+\kappa_{m} v_{2}\right), m \in Z_{2,1}^{B} \cup Z_{2,2}^{B} \\
b_{m 1} v_{1}+\max \left\{0, \kappa_{m} v_{2}-\eta_{m} v_{1}\right\}, m \in Z_{2,3}^{B},
\end{array}\right.
$$

we get that

$$
\begin{aligned}
\sum_{m \in Z_{2}^{B}} \max _{d}\left\{b_{g(m), d}^{*} v_{d}\right\}= & \sum_{m \in Z_{2}^{B}} b_{m 1} v_{1} \\
& +\sum_{m \in Z_{2,1}^{B} \cup Z_{2,2}^{B}}\left(b_{m 2} v_{2}-b_{m 1} v_{1}+\kappa_{m} v_{2}\right) \\
& +\sum_{m \in Z_{2,3}^{B}} \max \left\{0, \kappa_{m} v_{2}-\eta_{m} v_{1}\right\}
\end{aligned}
$$

By definition,

$$
l(\boldsymbol{\theta} \boldsymbol{v} \mid B)=\sum_{m \in Z_{2}^{B}} \max _{d}\left\{b_{g(m), d}^{*} v_{d}\right\}+\sum_{j \in I \backslash\left\{g(m): m \in Z_{2}^{B}\right\}} \max _{d}\left\{b_{j d}^{*} v_{d}\right\} .
$$

Now,

$$
\begin{aligned}
& l(\boldsymbol{\theta} \boldsymbol{v} \mid B)-l(\boldsymbol{\theta} \boldsymbol{v} \mid C)=l(\boldsymbol{\theta} \boldsymbol{v} \mid B)-\theta_{1} v_{1} \\
& =l(\boldsymbol{\theta} \boldsymbol{v} \mid B)-\sum_{m \in Z_{2}^{B}} b_{m 1} v_{1}-\sum_{j \in I \backslash\left\{g(m): m \in Z_{2}^{B}\right\}} b_{j 1}^{*} v_{1} \\
& \geq \sum_{m \in Z_{2,1}^{B} \cup Z_{2,2}^{B}}\left(b_{m 2} v_{2}-b_{m 1} v_{1}\right)
\end{aligned}
$$


where Eq. 40 holds with Eq. 34, Eq. 41 holds with Eq. 14, and Ineq. 42 follows from Eqs. 39 and 38. Finally, by Ineq. 37 and the argumentation at the beginning of part (2) we have that $\theta_{\text {inf }}(\boldsymbol{v})=\theta(\boldsymbol{v} \mid C)$.

(4) For $v_{1} \in V_{3}$ consider the process $C\left(\theta_{1}, \theta_{2}, \tilde{\phi}, v_{1}\right)$ in Example 3 and apply the same argumentation as in part (1).

Finally, we consider the case where $\phi$ instead of $\tilde{\phi}$ is given, cf. Fig. 4 .

Theorem 9 Let $D=2$ and $\Theta\left(\theta_{1}, \theta_{2}, \phi\right)$ be the closed set of multivariate extremal index functions $\theta$ for all stationary max-stable processes with univariate extremal indices $\theta_{1}, \theta_{2} \in(0,1]$ and adjusted extremal coefficient $\phi=l(\boldsymbol{\theta} \mathbf{1})=\tilde{\phi} \theta(\mathbf{1})$. Let $\theta_{\text {inf }}$ and $\theta_{\text {sup }}$ be defined analogously to Theorems 7 and 8. Then,

$$
\begin{aligned}
\theta_{\text {sup }}(\boldsymbol{v}) & =\left(1+\frac{\max _{d}\left(1-\theta_{d}\right) v_{d}}{\sum_{d}\left(\phi-\theta_{3-d}\right) v_{d}+\left(\theta_{1}+\theta_{2}-\phi\right) \max _{d} v_{d}}\right)^{-1}, \\
\theta_{\text {inf }}(\boldsymbol{v}) & =\min _{d}\left\{\max \left\{\theta_{d}-2\left(\theta_{d}-\phi / 2\right) v_{3-d}, \theta_{3-d} v_{3-d}\right\}\right\} .
\end{aligned}
$$

Further, $\theta_{\text {sup }} \in \Theta$ for all $\phi$, and $\theta_{\text {inf }} \notin \Theta$ iff $\max _{d} \theta_{d}<\phi<\sum_{d} \theta_{d}$.

Proof Let $\mathcal{B}=\mathcal{B}\left(\theta_{1}, \theta_{2}, \phi\right)$ be the class of $R S$ processes $B$ with coefficients $b_{j k d}, j \in I, k \in \mathbb{Z}, d=1,2$, and $\beta_{j k d}, j \in F, 0 \leq k \leq N_{j}, d=1,2$, such that $l\left(\boldsymbol{\theta} \boldsymbol{e}_{d} \mid B\right)=\theta_{d}$ and $l(\boldsymbol{\theta 1} \mid B)=\phi$.

The equality for $\theta_{\text {sup }}$ follows by Example 2 and the fact that $\mathcal{B} \subseteq \mathcal{X}\left(\theta_{1}, \theta_{2}, \phi\right)$, where $X^{*}\left(\theta_{1}, \theta_{2}, \phi\right) \in \mathcal{B}$.

To show the equality for $\theta_{\text {inf }}$ note that from $l(\boldsymbol{\theta 1} \mid B)=\phi$ it holds by (L1) and (L2) that

$$
\begin{aligned}
l(\boldsymbol{\theta} \boldsymbol{v} \mid B) & \geq \min _{d}\left\{\max \left\{\theta_{d}-2\left(\theta_{d}-\phi / 2\right) v_{3-d}, \theta_{3-d} v_{3-d}\right\}\right\} \\
& =\min \left\{l_{1}(\boldsymbol{v}), l_{2}(\boldsymbol{v})\right\}=\theta_{\mathrm{inf}}(\boldsymbol{v}),
\end{aligned}
$$

where $l_{1}$ and $l_{2}$ are valid dependence functions, see below. Then, the last equation follows from Theorem 3 (3). Further, $\theta_{\text {inf }}$ is not a valid dependence function for $\max _{d} \theta_{d}<\phi<\sum_{d} \theta_{d}$ by lack of convexity, and $\theta_{\text {inf }}(\boldsymbol{v})=\sum_{d} \theta_{d} v_{d}$ for $\phi=\sum_{d} \theta_{d}$ and $\theta_{\text {inf }}(\boldsymbol{v})=\max _{d} \theta_{d} v_{d}$ for $\phi=\max _{d} \theta_{d}$.

Finally, note that $\theta_{\text {inf }}$ is reached piecewise by the $R S$ example processes $B_{m} \in \mathcal{B}, m=1,2$, where $b_{m, 11 m}=\phi-\theta_{3-m}, b_{m, 21 m}=\sum_{d} \theta_{d}-\phi, b_{m,(2,2,3-m)}=$ $\theta_{3-m}, \beta_{m, 1 d d}=1-\theta_{d}$.

Acknowledgements The authors wish to thank the referees and Zakhar Kabluchko for valuable hints and comments.

Open Access This article is distributed under the terms of the Creative Common Attribution Noncommercial License which permits any noncommercial use, distribution, and reproduction in any medium, provided the original author(s) and source are credited. 


\section{References}

Beirlant, J., Goegebeur, Y., Segers, J., Teugels, J.: Statistics of Extremes. John Wiley \& Sons Ltd., Chichester (2004)

Deheuvels, P.: Point processes and multivariate extreme values. J. Multivar. Anal. 13, 257-272 (1983)

de Haan, L., Pickands, J.: Stationary min-stable stochastic processes. Probab. Th. Rel. Fields 72, 477-492 (1986)

Huang, X.: Statistics of bivariate extreme values. Ph.D. thesis, Erasmus University, Rotterdam (1992)

Krengel, U.: Ergodic Theorems. de Gruyter, Berlin (1985)

Leadbetter, M.R.: Extremes and local dependence of stationary sequences. Z. Wahrscheinlichkeitstheorie verw. Gebiete 65, 291-306 (1983)

Martins, A.P., Ferreira, H.: Measuring the extremal dependence. Stat. Prob. Lett. 73, 99-103 (2005a)

Martins, A.P., Ferreira, H.: The multivariate extremal index and the dependence structure of a multivariate extreme value distribution. Test 14, 433-448 (2005b)

Nandagopalan, S.: On the multivariate extremal index. J. Res. Natl. Inst. Stand. Technol. 99, 543-550 (1994)

Perfekt, R.: Extreme value theory for a class of markov chains with values in $\mathbb{R}^{d}$. Adv. Appl. Probab. 29, 138-164 (1997)

Pickands, J.: Multivariate extreme value distributions. Bull. Int. Stat. Inst. 49, 859-878 (1981)

Resnick, S.I.: Extreme Values, Regular Variation, and Point Processes. Springer, New York, Berlin (1987)

Samorodnitsky, G.: Extreme value theory, ergodic theory and the boundary between short memory and long memory for stationary stable processes. Ann. Probab. 32, 1438-1468 (2004)

Schlather, M., Tawn, J.A.: Inequalities for the extremal coefficients of multivariate extreme value distributions. Extremes 5, 87-102 (2002)

Schlather, M., Tawn, J.A.: A dependence measure for multivariate and spatial extreme values: Properties and inference. Biometrika 90(1), 139-156 (2003)

Smith, R.L.: Max-stable processes and spatial extremes. Unpublished manuscript (1990)

Smith, R.L., Weissman, I.: Characterization and estimation of the multivariate extremal index. Technical report, University of North Carolina at Chapel Hill, NC, USA (1996)

Stoev, S.A.: On the ergodicity and mixing of max-stable processes. Technical Report 461, Department of Statistics, University of Michigan (2007) 